\title{
A NUMERICAL SCHEME FOR THE PORE SCALE SIMULATION OF CRYSTAL DISSOLUTION AND PRECIPITATION IN POROUS MEDIA
}

\author{
V. M. DEVIGNE ${ }^{\natural, b}$, I. S. POP $\sharp$ C. J. VAN DUIJN ${ }^{\sharp}$, AND T. CLOPEAU
}

\begin{abstract}
In this paper we analyze a numerical scheme for a dissolution and precipitation in porous media. We focus here on the chemistry, which is modeled by a parabolic problem that is coupled through the boundary conditions to an ordinary differential inclusion defined on the boundary. We use a regularization approach for constructing a semi-implicit scheme that is stable and convergent. For dealing with the emerging time discrete nonlinear problems, we propose a simple fixed point iterative procedure. The paper is concluded by numerical results.
\end{abstract}

\section{INTRODUCTION}

In this paper we consider a pore scale model for the dissolution and precipitation of crystals in a porous medium. This model is studied in [4] and represents the pore-scale analogue of the macroscopic (core scale) model proposed in [12]. The particularity of the model is in the description of the precipitation processes taking place on the surface of the grains $\Gamma_{G}$, involving a multi valued function. Models of similar type are analyzed in a homogenization context in $[9,10,17]$.

Without going into details, we briefly recall the background of the model. A fluid in which cations and anions are dissolved occupies the pores of a porous medium. The boundary of the void space consists of two disjoint parts: the surface of the porous skeleton (the grains), named from now one the internal boundary, and the external part, which is the outer boundary of the domain. Under certain conditions, the ions transported by the fluid can precipitate and form a crystalline solid, which is attached to the internal boundary and thus is immobile. The reverse reaction of dissolution is also possible.

The model proposed in [4] consists of several components: the Stokes flow in the pores, the transport of dissolved ions by convection and diffusion, and dissolution/precipitation reactions on the surface of the porous skeleton (grains). It is assumed that the flow geometry, as well as the fluid properties are not affected by the chemical processes. 
Therefore the flow component can be completely decoupled from the remaining part of the model.

Another simplification follows by considering the total electric charge in the fluid. This is a linear combination of the concentrations of the ions, and can be determined by solving a linear parabolic problem that depends only on the flow and not on the chemistry. Therefore once the fluid flow is determined, the total charge can be decoupled from the chemistry (see [3] for details).

Our main interest is focused on the chemistry, this being the challenging part of the model. Here we investigate an appropriate numerical scheme for the dissolution and precipitation component of the model in [4]. The present work is closely related to [8]. The numerical scheme proposed there involves also a special treatment of the diffusion on the grain boundary. The coupled system is solved by iterating between the equations in the pores, and on the solid matrix.

Numerical methods for the macro scale equations modeling the dissolution and precipitation in porous media are considered in $[1,5,6$, 15, 16]. The time stepping is performed by various first order implicit schemes, and finite elements or finite volumes are employed for the spatial discretization. For the upscaled version of the present model, a numerical algorithm for computing traveling wave solutions is proposed in $[12]$.

We denote the flow domain by $\Omega \subset \mathbb{R}^{d}(d>1)$, which is assumed open, connected and bounded. Further, the boundary $\partial \Omega$ is assumed Lipschitz continuous. It consists of two disjoint parts: an internal and an external one. The internal boundary $\Gamma_{G}$ is the surface of the grains, and the external boundary $\Gamma_{D}$ is the outer boundary of the domain. Let $\vec{\nu}$ denote the outer normal to $\partial \Omega$ and $T>0$ be a fixed but arbitrarily chosen value of time. For $X$ being $\Omega, \Gamma_{G}$, or $\Gamma_{D}$, and any $0<t \leq T$, we define

$$
X^{t}=(0, t] \times X
$$

To simplify the presentation, we assume that the initial data are compatible in the sense of [3] and [12]. Essentially this means that initially the system is in equilibrium. Moreover, the boundary data are assumed such that the total charge remains constant in time and space. In this way, and after an appropriate scaling, the model can be reduced to

$$
\left\{\begin{aligned}
\partial_{t} u+\nabla \cdot(\overrightarrow{q u}-D \nabla u) & =0, & & \text { in } \Omega^{T}, \\
-D \vec{\nu} \cdot \nabla u & =\varepsilon \tilde{n} \partial_{t} v, & & \text { on } \Gamma_{G}^{T}, \\
u & =0, & & \text { on } \Gamma_{D}^{T}, \\
u & =u_{I}, & & \text { in } \Omega, \text { for } t=0,
\end{aligned}\right.
$$


for the ion transport, and

$$
\left\{\begin{aligned}
\partial_{t} v & =D_{a}(r(u)-w), & & \text { on } \Gamma_{G}^{T}, \\
w & \in H(v), & & \text { on } \Gamma_{G}^{T}, \\
v & =v_{I}, & & \text { on } \Gamma_{G}, \text { for } t=0,
\end{aligned}\right.
$$

for the precipitation and dissolution. Here $u$ denotes the cation concentration and is defined in the entire void space $\Omega$, whereas $v$ stands for the concentration of the precipitate, which is defined only on the interior boundary $\Gamma_{G}$. These two concentrations, together with $w$ modeling the actual value of the dissolution rate, are the unknown quantities.

In the above $\vec{q}$ is the divergence free fluid velocity, which is assumed known and having a zero trace along the internal grain boundary $\Gamma_{G}$ :

$$
\vec{q} \in\left[H^{1}(\Omega)\right]^{d}, \quad \nabla \cdot \vec{q}=0 \text { in } \Omega, \quad \vec{q}=\overrightarrow{0} \text { on } \Gamma_{G} .
$$

By $H^{1}(\Omega)$ we mean the space of functions defined on $\Omega$ and having $L^{2}$ generalized derivatives. To avoid unnecessary technical complications we also assume that the fluid velocity $\vec{q}$ is essentially bounded, $\vec{q} \in$ $\left[L^{\infty}(\Omega)\right]^{d}$, and define

$$
M_{q}=\|\vec{q}\|_{\infty, \Omega}<\infty .
$$

For the Stokes model with homogeneous Dirichlet boundary conditions, the essential boundedness of $\vec{q}$ holds if, for example, the domain is polygonal (see [11] or [14]).

The above equations are employing several functions, which are model specific. Here we assume them given. By $r$ we denote the precipitation rate. Assuming mass action kinetics, with $[\cdot]_{+}$denoting the nonnegative cut,

$$
[u]_{+}= \begin{cases}0, & \text { if } u<0, \\ u, & \text { if } u>0,\end{cases}
$$

the precipitation rate is defined by

$$
r(u)=[u]_{+}^{\tilde{m}}[(\tilde{m} u-c) / \tilde{n}]_{+}^{\tilde{n}},
$$

where $\tilde{m}$ and $\tilde{n}$ are the valences of the cation, respectively anion. As mentioned before, the total negative charge $c$ is assumed known and constant in time and space. In a generalized framework, $r$ satisfies the following:

$\left(\mathrm{A}_{r}\right) \quad$ (i) $r: \mathbb{R} \rightarrow[0, \infty)$ is locally Lipschitz in $\mathbb{R}$;

(ii) there exists a unique $u_{*} \geq 0$, such that

$$
r(u)=\left\{\begin{array}{l}
0, \quad \text { for } \quad u \leq u_{*}, \\
\text { strictly increasing for } u>u_{*} \text { with } r(\infty)=\infty ;
\end{array}\right.
$$


Remark 1.1. In this setting, a unique $u^{*}$ exists for which $r\left(u^{*}\right)=1$. If $u=u^{*}$ for all $t$ and $x$, then the system is in equilibrium: no precipitation or dissolution occurs, since the precipitation rate is balanced by the dissolution rate regardless of the presence or absence of crystals.

In $\left(1.2_{2}\right), H$ stands for the Heaviside graph,

$$
H(u)= \begin{cases}\{0\}, & \text { if } u<0 \\ {[0,1],} & \text { if } u=0 \\ \{1\}, & \text { if } u>0\end{cases}
$$

This implies that the dissolution rate is constant (scaled to 1 ) in the presence of crystal, i.e. for $v>0$ somewhere on $\Gamma_{G}$. In the absence of crystals, the overall rate is either zero if $r(u) \leq 1$, meaning that the fluid is not containing sufficient dissolved ions, or positive. This last situation is appearing in the oversaturated regime, $w=r(u)-1>0$.

Finally, $D$ in (1.1)-(1.2) denotes the diffusion coefficient. $D_{a}$ represents the ratio of the characteristic precipitation/dissolution time scale and the characteristic transport time scale - the Damköhler number. Both $D$ and $D_{a}$ are assumed to be of moderate order, $O(1)$. By $\varepsilon$ we mean the ratio of the characteristic pore scale and the reference (macroscopic) length scale. In an appropriate scaling (see Remark 1.2 of [4]), this gives

$$
\varepsilon \operatorname{meas}\left(\Gamma_{G}\right) \approx \operatorname{meas}(\Omega)
$$

This balance is natural for a porous medium, where meas $\left(\Gamma_{G}\right)$ denotes the total surface of the porous skeleton and meas $(\Omega)$ the total void volume. Throughout this paper we keep the value of $\varepsilon$ fixed. However, the convergence results are uniform with respect to $\varepsilon$, thus also for arbitrarily small values of $\varepsilon$.

The present setting is a simplification of the dissolution/precipitation model in [4]. However, the main difficulties that are associated with that model are still present here: the system (1.1)-(1.2) is consisting of a parabolic equation that is coupled through the boundary conditions to a differential inclusion defined on a lower dimensional manifold. For the ease of presentation we have considered here only the case of homogeneous Dirichlet boundary conditions on the external boundary $\Gamma_{D}$, but the results can be extended to more general cases. With $H_{0, \Gamma_{D}}^{1}(\Omega)$ being the subspace of $H^{1}(\Omega)$ functions having a zero trace on the external boundary $\Gamma_{D}$, for the initial data we assume

$\left(A_{D}\right) \quad$ The initial data are assumed essentially bounded and nonnegative. Further, $u_{I} \in H_{0, \Gamma_{D}}^{1}(\Omega)$ and $v_{I} \in L^{2}\left(\Gamma_{G}\right)$. 
Due to the occurrence of the multi-valued dissolution rate, classical solutions do not exists, except for some particular cases. For defining a weak solution we consider the following sets

$$
\begin{aligned}
\mathcal{U} & :=\left\{u \in L^{2}\left(0, T ; H_{0, \Gamma_{D}}^{1}(\Omega)\right): \partial_{t} u \in L^{2}\left(0, T ; H^{-1}(\Omega)\right)\right\} \\
\mathcal{V} & :=\left\{v \in H^{1}\left(0, T ; L^{2}\left(\Gamma_{G}\right)\right)\right\} \\
\mathcal{W} & :=\left\{w \in L^{\infty}\left(\Gamma_{G}^{T}\right),: 0 \leq w \leq 1\right\} .
\end{aligned}
$$

Here we have used standard notations in the functional analysis. In what follows $(\cdot, \cdot)_{X}$ stands for the usual scalar product in a Hilbert space $X$, or the duality pairing between $H^{-1}$ and $H^{1}$. With $t \in(0, T]$, by $(\cdot, \cdot)_{X^{t}}$ we mean the integration in time of $(\cdot, \cdot)_{X}$ on $(0, t)$.

Definition 1.1. A triple $(u, v, w) \in \mathcal{U} \times \mathcal{V} \times \mathcal{W}$ is called a weak solution of (1.1) and (1.2) if $\{u(0), v(0)\}=\left\{u_{I}, v_{I}\right\}$ and if

$$
\begin{aligned}
\left(\partial_{t} u, \varphi\right)_{\Omega^{T}}+D(\nabla u, \nabla \varphi)_{\Omega^{T}}-(\vec{q} u, \nabla \varphi)_{\Omega^{T}}=-\varepsilon \tilde{n}\left(\partial_{t} v, \varphi\right)_{\Gamma_{G}^{T}} & \\
\left(\partial_{t} v, \theta\right)_{\Gamma_{G}^{T}} & =D_{a}(r(u)-w, \theta)_{\Gamma_{G}^{T}}, \\
w & \in H(v) \quad \text { a.e. in } \Gamma_{G}^{T},
\end{aligned}
$$

for all $(\varphi, \theta) \in L^{2}\left(0, T ; H_{0, \Gamma_{D}}^{1}(\Omega)\right) \times L^{2}\left(\Gamma_{G}^{T}\right)$.

In the above definition the initial condition should be understood in the sense of $L^{2}$ functions. This makes sense since $\mathcal{U} \subset C\left([0, T] ; L^{2}(\Omega)\right)$, respectively $\mathcal{V} \subset C\left([0, T] ; L^{2}\left(\Gamma_{G}\right)\right.$ ) (see for example [22], or [26]). The existence of a weak solution is proven in [4], Theorem 2.21. Moreover, with

$$
\begin{aligned}
& M_{u}:=\max \left\{\left\|u_{I}\right\|_{\infty, \Omega}, u^{*}\right\}, \\
& M_{v}:=\max \left\{\left\|v_{I}\right\|_{\infty, \Omega}, 1\right\}, \quad C_{v}:=\frac{r\left(M_{u}\right) D_{a}}{M_{v}},
\end{aligned}
$$

a weak solution satisfies

$$
\begin{aligned}
0 \leq u \leq M_{u}, & \text { a.e. in } \Omega^{T} \\
0 \leq v(t, \cdot) \leq M_{v} e^{C_{v} t}, & \text { for all } t \in[0, T] \text { and a.e. on } \Gamma_{G}, \\
0 \leq w \leq 1, & \text { a.e. on } \Gamma_{G}^{T},
\end{aligned}
$$

and

$$
\begin{aligned}
&\|u(t)\|_{\Omega}^{2}+\|\nabla u\|_{\Omega^{T}}^{2}+\left\|\partial_{t} u\right\|_{L^{2}\left(0, T ; H^{-1}(\Omega)\right)}^{2} \\
&+\varepsilon\|v(t)\|_{\Gamma_{G}}^{2}+\varepsilon\left\|\partial_{t} v\right\|_{\Gamma_{G}^{T}}^{2} \leq C,
\end{aligned}
$$


for all $0 \leq t \leq T$. Here $C>0$ is a constant not depending on $u, v, w$, or $\varepsilon$. The proof is based on regularization arguments and provides a solution for which, in addition, we have

$$
w=r(u) \quad \text { a.e. in }\{v=0\} \cap \Gamma_{G}^{T} \text {. }
$$

\section{The time Discrete numerical SCHEME}

In this section we analyze a semi-implicit numerical scheme for the system (1.1)-(1.2). To overcome the difficulties that are due to the multi-valued dissolution rate we approximate the Heaviside graph by

$$
H_{\delta}(v):= \begin{cases}0, & \text { if } v \leq 0, \\ v / \delta, & \text { if } v \in(0, \delta), \\ 1, & \text { if } v \geq \delta,\end{cases}
$$

where $\delta>0$ is a small regularization parameter.

Next we consider a time stepping that is implicit in $u$ and explicit in $v$. Though possible here as well, an implicit discretization of $v$ would involve an additional nonlinearity in $v$ without bringing any significant improvement of the results.

With $N \in \mathbb{N}, \tau=T / N$, and $t_{n}=n \tau(n=0, \ldots, N)$, the approximation pair $\left(u^{n}, v^{n}\right)$ of $\left(u\left(t_{n}\right), v\left(t_{n}\right)\right)$ is the solution of the following problem:

Problem $\mathbf{P}_{\delta}^{n}$ : Given $u^{n-1}, v^{n-1}$, compute $u^{n} \in H_{0, \Gamma_{D}}^{1}(\Omega)$, and $v^{n} \in$ $L^{2}\left(\Gamma_{G}\right)$ such that

$$
\begin{aligned}
&\left(u^{n}-u^{n-1}, \phi\right)_{\Omega}+\tau D\left(\nabla u^{n}, \nabla \phi\right)_{\Omega}-\tau\left(\vec{q} u^{n}, \nabla \phi\right)_{\Omega} \\
&+\epsilon \tilde{n}\left(v^{n}-v^{n-1}, \phi\right)_{\Gamma_{G}}=0, \\
&\left(v^{n}, \theta\right)_{\Gamma_{G}}=\left(v^{n-1}, \theta\right)_{\Gamma_{G}}+ \tau D_{a}\left(r\left(u^{n}\right)-H_{\delta}\left(v^{n-1}\right), \theta\right)_{\Gamma_{G}},
\end{aligned}
$$

for all $\phi \in H_{0, \Gamma_{D}}^{1}(\Omega)$ and $\theta \in L^{2}\left(\Gamma_{G}\right)$.

Here $n=1, \ldots, N$, while $u^{0}=u_{I}$ and $v^{0}=v_{I}$. For the consistency with the original setting, in (2.3) we approximate the dissolution rate $w\left(t_{n}\right)$ by

$$
w^{n}:=H_{\delta}\left(v^{n}\right) .
$$

To simplify the notations, we have given up the subscript $\delta$ for the solution triple $\left(u^{n}, v^{n}, w^{n}\right)$.

Remark 2.1. As we will see later, for guaranteeing the stability of the scheme the regularization parameter $\delta$ should be chosen such that 
$\delta \geq \tau D_{a}$. This is the only restriction that is related to the explicit discretization of $v$. Further, the convergence result is obtained assuming $\delta=O\left(\tau^{\alpha}\right)$ with some $\alpha \in(0,1)$, which is consistent with the previous restriction.

Due to the explicit discretization of $v$, the ion transport equation in Problem $\mathrm{P}_{\delta}^{n}$ can be decoupled from the time discrete precipitation/dissolution equation. Replacing the $\Gamma_{G}$-scalar product in $(2.2)$ by the last term in (2.3), we end up with an elliptic problem having a nonlinear boundary condition on $\Gamma_{G}$. Specifically, given $u^{n-1}$ and $v^{n-1}$, we seek for $u^{n} \in H_{0, \Gamma_{D}}^{1}(\Omega)$ such that

$$
\begin{aligned}
\left(u^{n}-u^{n-1}, \phi\right)_{\Omega} & +\tau D\left(\nabla u^{n}, \nabla \phi\right)_{\Omega}+\tau\left(\nabla \cdot\left(\vec{q} u^{n}\right), \phi\right)_{\Omega} \\
& +\tau \epsilon \tilde{n} D_{a}\left(r\left(u^{n}\right)-H_{\delta}\left(v^{n-1}\right), \theta\right)_{\Gamma_{G}}=0,
\end{aligned}
$$

for all $\phi \in H_{0, \Gamma_{D}}^{1}(\Omega)$. Since $r$ is monotone and Lipschitz, standard monotonicity methods provide the existence and uniqueness for the above problem (see e.g [25], Chapter 10). Alternatively, a contraction argument is described in Section 3. This writing is used there as the starting point for constructing a linear iterative scheme for solving Problem $\mathrm{P}_{\delta}^{n}$. The fixed point approach proving the convergence of the iteration procedure discussed in Section 3 can also be employed for the existence and uniqueness of a $u^{n}$ solving the nonlinear problem above. Having $u^{n}, v^{n}$ can be determined straightforwardly from (2.3). In this way we obtain

Lemma 2.2. Problem $P_{\delta}^{n}$ has a unique solution pair $\left(u^{n}, v^{n}\right)$.

2.1. Stability in $L^{\infty}$. All the estimates in this section should be interpreted in the almost everywhere (a.e.) sense. As follows from (1.12), the concentrations $u$ and $v$, as well as the dissolution rate $w$ are nonnegative and bounded. Here we prove similar results for the time discrete concentrations $u^{n}$ and $v^{n}$. The bounds for $w^{n}$ follow straightforwardly from (2.4).

Lemma 2.3. Assume $\delta \geq \tau D_{a}$ and that $u^{n-1}$ and $v^{n-1}$ are nonnegative. Then $u^{n}$ and $v^{n}$ are non-negative as well.

Proof. We start with the estimate in $v^{n}$. With $[\cdot]_{-}$denoting the nonpositive cut (see also (1.5)), we test (2.3) with $\theta:=\left[v^{n}\right]_{-}$and obtain

$\left\|\left[v^{n}\right]_{-}\right\|_{\Gamma_{G}}^{2}=\tau D_{a}\left(r\left(u^{n}\right),\left[v^{n}\right]_{-}\right)_{\Gamma_{G}}+\left(v^{n-1}-\tau D_{a} H_{\delta}\left(v^{n-1}\right),\left[v^{n}\right]_{-}\right)_{\Gamma_{G}}$

In view of $\left(\mathrm{A}_{r}\right)$, the first term on the right is non-positive. Further, since $v^{n-1} \geq 0$ and $\delta \geq \tau D_{a}$, by the construction of $H_{\delta}$ we have

$$
v^{n-1}-\tau D_{a} H_{\delta}\left(v^{n-1}\right) \geq v^{n-1}\left(1-\tau D_{a} / \delta\right) \geq 0,
$$


almost everywhere on $\Gamma_{G}$. Hence the second term on the right is nonpositive as well. This yields

$$
\left\|\left[v^{n}\right]_{-}\right\|_{\Gamma_{G}}^{2} \leq 0
$$

implying the assertion for $v^{n}$.

For proving that $u^{n}$ is non-negative we proceed in a similar manner. Testing (2.2) with $\phi:=\left[u^{n}\right]_{-}$gives

$$
\begin{aligned}
\left\|\left[u^{n}\right]_{-}\right\|_{\Omega}^{2}+\tau D & \left\|\nabla\left[u^{n}\right]_{-}\right\|_{\Omega}^{2}-\tau\left(\vec{q} u^{n}, \nabla\left[u^{n}\right]_{-}\right)_{\Omega} \\
+ & \epsilon \tilde{n}\left(v^{n}-v^{n-1},\left[u^{n}\right]_{-}\right)_{\Gamma_{G}}=\left(u^{n-1},\left[u^{n}\right]_{-}\right)_{\Omega} .
\end{aligned}
$$

The first two terms in the above are non-negative, whereas the third one vanishes. This follows from

$$
\begin{aligned}
\left(\vec{q} u^{n}, \nabla\left[u^{n}\right]_{-}\right)_{\Omega} & =\frac{1}{2}\left(\vec{q}, \nabla\left[u^{n}\right]_{-}^{2}\right)_{\Omega} \\
& =\frac{1}{2}\left(\vec{\nu} \cdot \vec{q},\left[u^{n}\right]_{-}^{2}\right)_{\Gamma_{D} \cup \Gamma_{G}}-\frac{1}{2}\left(\nabla \cdot \vec{q},\left[u^{n}\right]_{-}^{2}\right)_{\Omega},
\end{aligned}
$$

and the boundary conditions on $\partial \Omega$, since $\nabla \cdot \vec{q}=0$ in $\Omega$.

Further, since $\left[u^{n}\right]_{-} \leq 0$ a.e. and it belongs to $H_{0, \Gamma_{D}}^{1}(\Omega)$, its trace $\left.\left[u^{n}\right]_{-}\right|_{\Gamma_{G}}$ is a non-positive $L^{2}\left(\Gamma_{G}\right)$ function. Testing (2.3) with $\left.\left[u^{n}\right]_{-}\right|_{\Gamma_{G}}$ gives

$$
\begin{aligned}
\left(v^{n}-v^{n-1},\left[u^{n}\right]_{-}\right)_{\Gamma_{G}} & =\tau D_{a}\left(r\left(u^{n}\right)-H_{\delta}\left(v^{n-1}\right),\left[u^{n}\right]_{-}\right)_{\Gamma_{G}} \\
& =-\tau D_{a}\left(H_{\delta}\left(v^{n-1}\right),\left[u^{n}\right]_{-}\right)_{\Gamma_{G}} \geq 0
\end{aligned}
$$

where we have used $\left(\mathrm{A}_{r}\right)$ and the positivity of $H_{\delta}$.

Finally, the term on the right in (2.5) is non-positive, since $u^{n-1} \geq 0$. In this way we obtain $\left[u^{n}\right]_{-}=0$ almost everywhere in $\Omega$, implying the result.

Now we turn our attention to the upper bounds for $u^{n}$ and $v^{n}$. First, with $M_{u}$ defined in (1.10) we have

Lemma 2.4. If $u^{n-1} \leq M_{u}$, then the same holds for $u^{n}$.

Proof. We test $(2.2)$ with $\phi:=\left[u^{n}-M_{u}\right]_{+}$, the non-negative part of $u^{n}-M_{u}$. This gives

$$
\begin{aligned}
& \left\|\left[u^{n}-M_{u}\right]_{+}\right\|_{\Omega}^{2}+\tau D\left\|\nabla\left[u^{n}-M_{u}\right]_{+}\right\|_{\Omega}^{2}-\tau\left(\vec{q} u^{n}, \nabla\left[u^{n}-M_{u}\right]_{+}\right)_{\Omega} \\
& \quad=\left(u^{n-1}-M_{u},\left[u^{n}-M_{u}\right]_{+}\right)_{\Omega}-\epsilon \tilde{n}\left(v^{n}-v^{n-1},\left[u^{n}-M_{u}\right]_{+}\right)_{\Gamma_{G}} .
\end{aligned}
$$

Arguing as in the proof of Lemma 2.3, we first observe that the convection term vanishes. Further, since $u^{n-1} \leq M_{u}$, the first term on the right is non-positive. Finally, for the last term we have

$$
\left(v^{n}-v^{n-1},\left[u^{n}-M_{u}\right]_{+}\right)_{\Gamma_{G}}=\tau D_{a}\left(r\left(u^{n}\right)-H_{\delta}\left(v^{n-1}\right),\left[u^{n}-M_{u}\right]_{+}\right)_{\Gamma_{G}} .
$$


By the definition of $M_{u}$, whenever $u^{n} \geq M_{u}$ we have $r\left(u^{n}\right) \geq 1 \geq$ $H_{\delta}\left(v^{n-1}\right)$. This implies the positivity of the above scalar product. We are therefore left with

$$
\left\|\left[u^{n}-M_{u}\right]_{+}\right\|_{\Omega}^{2}+\tau D\left\|\nabla\left[u^{n}-M_{u}\right]_{+}\right\|_{\Omega}^{2} \leq 0
$$

implying that $u^{n} \leq M_{u}$.

Remark 2.5. Since for the initial data we assume $0 \leq u_{I} \leq M_{u}$, and $0 \leq v_{I}$, Lemmata 2.3 and 2.4 show that $0 \leq u^{n} \leq M_{u}$ and $0 \leq v^{n}$ for all $n=0, \ldots, N$. Further, notice that the restriction $\delta \geq \tau D_{a}$ only appears due to the explicit treatment of $v$ in (2.3). As stated in Remark 2.1, a fully implicit scheme would make it unnecessary.

Upper estimates for $v^{n}$ can be obtained as for $u^{n}$.

Lemma 2.6. With $M_{v}$ and $C_{v}$ defined in (1.11), assume that $v^{n-1} \leq$ $M_{v} e^{C_{v}(n-1) \tau}$. Then $v^{n} \leq M_{v} e^{C_{v} n \tau}$.

Proof. Testing (2.3) with $\theta:=\left[v^{n}-M_{v} e^{C_{v} n \tau}\right]+$ gives

$$
\begin{gathered}
\left\|\left[v^{n}-M_{v} e^{C_{v} n \tau}\right]_{+}\right\|_{\Gamma_{G}}^{2}=\left(v^{n-1}-M_{v} e^{C_{v}(n-1) \tau},\left[v^{n}-M_{v} e^{C_{v} n \tau}\right]_{+}\right)_{\Gamma_{G}} \\
+M_{v}\left(e^{C_{v}(n-1) \tau}-e^{C_{v} n \tau},\left[v^{n}-M_{v} e^{C_{v} n \tau}\right]_{+}\right)_{\Gamma_{G}} \\
+\tau D_{a}\left(r\left(u^{n}\right)-H_{\delta}\left(v^{n-1}\right),\left[v^{n}-M_{v} e^{C_{v} n \tau}\right]_{+}\right)_{\Gamma_{G}}
\end{gathered}
$$

We denote the terms on the right by $I_{1}, I_{2}$ and $I_{3}$. We first notice that the assumption on $v^{n-1}$ imply $I_{1} \leq 0$. Further, since $0 \leq u^{n} \leq M_{u}$, $H_{\delta}\left(v^{n-1}\right) \geq 0$, and due to the monotonicity of $r$ we obtain

$$
I_{3} \leq \tau D_{a}\left(r\left(M_{u}\right),\left[v^{n}-M_{v} e^{C_{v} n \tau}\right]_{+}\right)_{\Gamma_{G}} .
$$

Recalling (1.11), this gives

$$
I_{2}+I_{3} \leq M_{v}\left(\tau C_{v}+e^{C_{v}(n-1) \tau}\left(1-e^{\tau C_{v}}\right),\left[v^{n}-M_{v} e^{C n \tau}\right]_{+}\right)_{\Gamma_{G}} \leq 0 .
$$

Here we have used the elementary inequality $e^{x} \geq 1+x$, as well as $e^{C_{v}(n-1) \tau} \geq 1$. In this way (2.6) becomes

$$
\left\|\left[v^{n}-M_{v} e^{C_{v} n \tau}\right]_{+}\right\|_{\Gamma_{G}}^{2} \leq 0
$$

implying the upper bounds for $v^{n}$.

Remark 2.7. As before, since $v_{I} \leq M_{v}$, it follows that $v^{n} \leq M_{v} e^{C n \tau}$ for all $n=1, \ldots, N$.

Remark 2.8. The essential bounds provided by Lemmata 2.3, 2.4 and 2.6 are uniform in $\delta$, assuming that $\delta \geq \tau D_{a}$. Moreover, $\tau$ only appears in the upper bounds for $v^{n}$. But for any $0 \leq n \leq N$ one has $n \tau=t_{n} \leq$ $T$, and we have $v^{n} \leq M_{v} e^{C_{v} t_{n}} \leq M_{v} e^{C_{v} T}$, so $\tau$-independent estimates can be obtained as well. 
2.2. A priori estimates. We continue the analysis of the numerical scheme (2.2)-(2.3) by giving some energy estimates for the sequence of time discrete concentrations $\left\{\left(u^{n}, v^{n}\right), n=0, \ldots, N\right\}$. We start with the estimates in $v$, which are depending on meas $\left(\Gamma_{G}\right)$.

Lemma 2.9. For any $n \geq 1$ we have:

$$
\begin{aligned}
\left\|v^{n}-v^{n-1}\right\|_{\Gamma_{G}} & \leq \tau D_{a} r\left(M_{u}\right) \operatorname{meas}\left(\Gamma_{G}\right)^{1 / 2}, \quad \text { and } \\
\left\|v^{n}\right\|_{\Gamma_{G}} & \leq\left\|v_{I}\right\|_{\Gamma_{G}}+n \tau D_{a} r\left(M_{u}\right) \operatorname{meas}\left(\Gamma_{G}\right)^{1 / 2} .
\end{aligned}
$$

Proof. Testing (2.3) with $\theta:=\left[v^{n}-v^{n-1}\right] \in L^{2}\left(\Gamma_{G}\right)$ and applying Cauchy's inequality gives,

$$
\left\|v^{n}-v^{n-1}\right\|_{\Gamma_{G}}^{2} \leq \tau D_{a}\left\|r\left(u^{n}\right)-H_{\delta}\left(v^{n-1}\right)\right\|_{\Gamma_{G}}\left\|v^{n}-v^{n-1}\right\|_{\Gamma_{G}}
$$

The essential bounds on $u^{n}$ and $v^{n}$, together with the assumptions on $r$ and $H_{\delta}$ imply $-1 \leq r\left(u^{n}\right)-H_{\delta}\left(v^{n-1}\right) \leq r\left(M_{u}\right)$. By (1.10) we have $r\left(M_{u}\right) \geq 1$, implying the first estimates.

In a similar manner, taking $\theta:=v^{n} \in L^{2}\left(\Gamma_{G}\right)$ in (2.3) and applying the Cauchy inequality we obtain

$$
\left\|v^{n}\right\|_{\Gamma_{G}}^{2} \leq\left\|v^{n-1}\right\|_{\Gamma_{G}}\left\|v^{n}\right\|_{\Gamma_{G}}+\tau D_{a} r\left(M_{u}\right) \operatorname{meas}\left(\Gamma_{G}\right)^{1 / 2}\left\|v^{n}\right\|_{\Gamma_{G}} .
$$

Dividing by $\left\|v^{n}\right\|_{\Gamma_{G}}$ and applying the inequality backward we immediately obtain the estimate (2.8).

Remark 2.10. Notice that the estimates in Lemma 2.9 are $\delta$-independent. Next for $n \leq N$, the term on the right in (2.8) is bounded uniformly in $\tau$ as well. Finally, due to (1.7) we can replace meas $\left(\Gamma_{G}\right)$ by $C / \epsilon$, where $C$ does not depend on $\delta, \tau$, or $\epsilon$.

Now we proceed by the estimates for $u$.

Lemma 2.11. Assume $\tau \leq \tau_{0}$, with $\tau_{0}>0$ a fixed value that will be given below. For the time discrete solute concentrations we have

$$
\begin{aligned}
\tau \sum_{n=1}^{N}\left\|\nabla u^{n}\right\|_{\Omega}^{2} & \leq C \\
\sum_{n=1}^{N}\left\|u^{n}-u^{n-1}\right\|_{\Omega}^{2} & \leq C \sqrt{\tau} \\
\tau \sum_{n=1}^{N}\left\|\nabla\left(u^{n}-u^{n-1}\right)\right\|_{\Omega}^{2} & \leq C \sqrt{\tau} \\
\sum_{n=1}^{N}\left\|u^{n}-u^{n-1}\right\|_{\Gamma_{G}}^{2} & \leq C .
\end{aligned}
$$


Here $C$ is a constant that does not depend on $\delta$ and $\tau$.

Proof. We start by testing (2.2) with $\phi=u^{n}$. This gives

$$
\begin{aligned}
& \left(u^{n}-u^{n-1}, u^{n}\right)_{\Omega}+\tau D\left\|\nabla u^{n}\right\|_{\Omega}^{2} \\
& \quad+\tau\left(\vec{q} u^{n}, \nabla u^{n}\right)_{\Omega}+\varepsilon \tilde{n}\left(v^{n}-v^{n-1}, u^{n}\right)_{\Gamma_{G}}=0 .
\end{aligned}
$$

We denote the terms on the right by $T_{1}, \ldots, T_{4}$. We have

$$
T_{1}=\frac{1}{2}\left(\left\|u^{n}\right\|_{\Omega}^{2}-\left\|u^{n-1}\right\|_{\Omega}^{2}+\left\|u^{n}-u^{n-1}\right\|_{\Omega}^{2}\right) .
$$

Furthermore, $T_{2}$ is non-negative, and $T_{3}$ vanishes as argued in the proof of Lemma 2.3.

Before estimating the last term we notice the existence of positive constants $C_{1}$ and $C_{2}$ such that

$$
\|\varphi\|_{\Gamma_{G}}^{2} \leq C_{1}\|\varphi\|_{\Omega}^{2}+C_{2}\|\varphi\|_{\Omega}\|\nabla \varphi\|_{\Omega}
$$

for all $\varphi \in H_{0, \Gamma_{D}}^{1}(\Omega)$. This can be obtained by following the proof of the trace theorem (e.g. see [7], Theorem 1.5.1.10). By the inequality of means

$$
a b \leq \frac{1}{4 \delta} a^{2}+\delta b^{2}, \quad \text { for all } a, b, \text { and } \delta>0,
$$

we get

$$
\|\varphi\|_{\Gamma_{G}}^{2} \leq\left(C_{1}+\frac{C_{2}^{2}}{4 \delta}\right)\|\varphi\|_{\Omega}^{2}+\delta\|\nabla \varphi\|_{\Omega}^{2}
$$

Here $\delta>0$ can be taken arbitrarily small.

With $M:=\varepsilon \tilde{n} D_{a} r\left(M_{u}\right)$ meas $\left(\Gamma_{G}\right)^{1 / 2}$, we use (2.7) to estimate $T_{4}$ :

$$
\left|T_{4}\right| \leq \tau M\left\|u^{n}\right\|_{\Gamma_{G}} \leq \frac{\tau M^{2}}{4}+\tau\left\|u^{n}\right\|_{\Gamma_{G}}^{2}
$$

Now we use (2.18) with $\delta=D / 2$ and obtain

$$
\left|T_{4}\right| \leq \frac{\tau M^{2}}{4}+\tau\left(C_{1}+\frac{C_{2}^{2}}{2 D}\right)\left\|u^{n}\right\|_{\Omega}^{2}+\frac{\tau D}{2}\left\|\nabla u^{n}\right\|_{\Omega}^{2}
$$

Using the estimates above into (2.15), summing for $n=1, \ldots, N$ and multiplying the result by 2 yields

$$
\left\|u^{N}\right\|_{\Omega}^{2}+\sum_{n=1}^{N}\left\|u^{n}-u^{n-1}\right\|_{\Omega}^{2}+\tau D \sum_{n=1}^{N}\left\|\nabla u^{n}\right\|_{\Omega}^{2} \leq\left\|u_{I}\right\|_{\Omega}^{2}+\frac{T M^{2}}{2}+C,
$$

where $C=T\left(C_{1}+C_{2}^{2} /(2 D)\right) M_{u}^{2}$ meas $(\Omega)$. This estimate follows by the essential bounds on $u^{n}$. An alternative proof can be given based 
on the discrete Gronwall lemma. In this way we have proven (2.11). Notice that we have also obtained

$$
\sum_{n=1}^{N}\left\|u^{n}-u^{n-1}\right\|_{\Omega}^{2} \leq C,
$$

which is not so good as (2.12).

To proceed with (2.12) and (2.13) we notice that, since $u_{I} \in H_{0, \Gamma_{D}}^{1}$, $u^{n}-u^{n-1}$ is a $H_{0, \Gamma_{D}}^{1}$ function for all $n \geq 1$. Testing (2.2) with $u^{n}-u^{n-1}$ gives

$$
\begin{aligned}
& \left\|u^{n}-u^{n-1}\right\|_{\Omega}^{2}+\tau D\left(\nabla u^{n}, \nabla\left(u^{n}-u^{n-1}\right)\right)_{\Omega} \\
& \quad-\tau\left(\vec{q} u^{n}, \nabla\left(u^{n}-u^{n-1}\right)\right)_{\Omega}+\epsilon \tilde{n}\left(v^{n}-v^{n-1}, u^{n}-u^{n-1}\right)_{\Omega}=0 .
\end{aligned}
$$

Denoting the terms in the above by $I_{1}, \ldots, I_{4}$, we have

$$
I_{2}=\frac{\tau D}{2}\left(\left\|\nabla u^{n}\right\|_{\Omega}^{2}-\left\|\nabla u^{n-1}\right\|_{\Omega}^{2}+\left\|\nabla\left(u^{n}-u^{n-1}\right)\right\|_{\Omega}^{2}\right) .
$$

Recalling (1.4), the inequality of means gives

$$
\begin{aligned}
\left|I_{3}\right| & =\tau\left(\nabla \cdot\left(\vec{q} u^{n}\right), u^{n}-u^{n-1}\right)_{\Omega} \leq \tau M_{q}\left\|\nabla u^{n}\right\|_{\Omega}\left\|u^{n}-u^{n-1}\right\|_{\Omega} \\
& \leq \frac{1}{2}\left\|u^{n}-u^{n-1}\right\|_{\Omega}^{2}+\frac{\tau^{2} M_{q}^{2}}{2}\left\|\nabla u^{n}\right\|_{\Omega}^{2} .
\end{aligned}
$$

With $M$ defined above, for $I_{4}$ we use the estimate (2.7) and obtain

$$
\left|I_{4}\right| \leq \varepsilon \tilde{n}\left\|v^{n}-v^{n-1}\right\|_{\Gamma_{G}}\left\|u^{n}-u^{n-1}\right\|_{\Gamma_{G}} \leq \frac{(\tau M)^{2}}{4 \mu_{1}}+\mu_{1}\left\|u^{n}-u^{n-1}\right\|_{\Gamma_{G}}^{2},
$$

where $\mu_{1}>0$ will be chosen below. Now we can take $\rho=\tau D /\left(4 \mu_{1}\right)$ into (2.18) to obtain

$$
\left|I_{4}\right| \leq \frac{(\tau M)^{2}}{4 \mu_{1}}+\mu_{1}\left(C_{1}+\frac{\mu_{1} C_{2}^{2}}{\tau D}\right)\left\|u^{n}-u^{n-1}\right\|_{\Omega}^{2}+\frac{\tau D}{4}\left\|\nabla\left(u^{n}-u^{n-1}\right)\right\|_{\Omega}^{2} .
$$

Using the above estimates into (2.19), taking $\mu_{1}=\sqrt{\tau D} / C_{2}$, multiplying the result by 2 and summing up for $n=1, \ldots, N,(2.11)$ gives

$$
\begin{gathered}
\left(\frac{1}{2}-\frac{\sqrt{\tau D}}{C_{2}}\right) \sum_{n=1}^{N}\left\|u^{n}-u^{n-1}\right\|_{\Omega}^{2}+\frac{\tau D}{2} \sum_{n=1}^{N}\left\|\nabla\left(u^{n}-u^{n-1}\right)\right\|_{\Omega}^{2} \\
\leq \tau D\left\|\nabla u_{I}\right\|_{\Omega}^{2}+\frac{T M^{2} C_{2}}{\sqrt{D}} \tau^{1 / 2}+\tau C .
\end{gathered}
$$

For $\tau_{0}:=D C_{2}^{2} /\left(16 C_{1}^{2}\right)$ and $\tau \leq \tau_{0}$, since $u_{I} \in H_{0, \Gamma_{D}}^{1}$ the inequality above immediately implies (2.12) and (2.13).

Finally, using (2.18) with $\rho=\tau^{1 / 2},(2.14)$ is a direct consequence of (2.12) and (2.13). 
Remark 2.12. As in Remark 2.10, if the medium is $\epsilon$-periodic, the constant $C$ in Lemma 2.11 does not depend on $\epsilon$. To see this we recall (1.7), and Lemma 3 of [9], saying that there exists a constant $C>0$, independent of $\varepsilon$, such that

$$
\varepsilon\|\varphi\|_{\Gamma_{G}}^{2} \leq C\left(\|\varphi\|_{\Omega}^{2}+\varepsilon^{2}\|\nabla \varphi\|_{\Omega}^{2}\right)
$$

for all $\varphi \in H^{1}(\Omega)$. Then the boundary term in (2.19) yields a constant $C$ which does not depend on $\varepsilon$ as well.

2.3. Convergence. In this part we proceed by proving the convergence of the numerical scheme defined in (2.2)-(2.3) to a weak solution of the system (1.1)-(1.2), as given in Definition 1.1. The multivalued dissolution rate hinders us in obtaining useful error estimates. Therefore convergence will be achieved by compactness arguments. In doing so we mainly follow the ideas in [4].

Considering the sequence of time discrete triples $\left\{\left(u^{n}, v^{n}, w^{n}\right), n=\right.$ $1, \ldots, N\}$ solving the problems $\mathrm{P}_{\delta}^{n}$, where $w^{n}$ is defined in (2.4), we construct an approximation of the solution of (1.1) and (1.2) for all times $t \in[0, T]$. Specifically, define for any $n=1, \ldots, N$ and $t \in$ $\left(t_{n-1}, t_{n}\right]$

$$
Z^{\tau}(t):=z^{n} \frac{\left(t-t_{n-1}\right)}{\tau}+z^{n-1} \frac{\left(t_{n}-t\right)}{\tau},
$$

where $(z, Z)$ stands for $(u, U)$ or $(v, V)$, and define

$$
W^{\tau}(t):=H_{\delta}\left(V^{\tau}(t)\right)
$$

Notice that $U_{\tau}, V_{\tau}$, and $W_{\tau}$ do not only depend on $\tau$, but also on the regularization parameter $\delta$.

For the time continuous triple $\left.\left\{U^{\tau}, V^{\tau}, W^{\tau}\right)\right\}$ we can use the uniform $L^{\infty}$ bounds, as well as the a priori estimates in Lemmata 2.9 and 2.11 , to derive $\delta$-independent estimates that are similar to those for the solution defined in Definition 1.1 (see [4]).

Lemma 2.13. Assume $\delta \geq \tau D_{a}$. Then for $\left(U^{\tau}, V^{\tau}, W^{\tau}\right)$ we have:

$$
0 \leq U^{\tau} \leq M_{u}, \quad \text { a.e. in } \Omega^{T}
$$

$$
\begin{array}{r}
0 \leq V^{\tau} \leq M_{v} e^{C T}, 0 \leq W^{\tau} \leq 1, \quad \text { a.e. in } \Gamma_{G}^{T}, \\
\left\|U^{\tau}(t)\right\|_{\Omega}+\left\|V^{\tau}(t)\right\|_{\Gamma_{G}}^{2} \leq C, \quad \text { for all } 0 \leq t \leq T, \\
\left\|\partial_{t} U^{\tau}\right\|_{L^{2}\left(0, T ; H^{-1}(\Omega)\right)}^{2}+\left\|\nabla U^{\tau}\right\|_{\Omega^{T}}^{2}+\left\|\partial_{t} V^{\tau}\right\|_{\Gamma_{G}^{T}}^{2} \leq C .
\end{array}
$$

Here $C>0$ is a constant that does not depend on $\tau$ or $\delta$. 
Proof. The essential bounds in (2.22) and (2.23) are a direct consequence of Lemmata 2.3, 2.4, 2.6, and of (2.4). The same holds for (2.24), for which we employ the stability estimates in Lemmata 2.9 and 2.11 .

To proceed with the gradient estimates in $(2.25)$ we notice that

$$
\begin{aligned}
\int_{0}^{T}\left\|\nabla U^{\tau}(t)\right\|_{\Omega}^{2} d t \leq & 2 \sum_{n=1}^{N} \tau\left\|\nabla u^{n-1}\right\|_{\Omega}^{2} \\
& +\int_{t_{n-1}}^{t_{n}} 2 \frac{\left(t-t_{n-1}\right)^{2}}{\tau^{2}}\left\|\nabla\left(u^{n}-u^{n-1}\right)\right\|_{\Omega}^{2} d t \\
\leq & 2 \tau \sum_{n=1}^{N}\left\|\nabla u^{n-1}\right\|_{\Omega}^{2}+\frac{2 \tau}{3} \sum_{n=1}^{N}\left\|\nabla\left(u^{n}-u^{n-1}\right)\right\|_{\Omega}^{2} \leq C .
\end{aligned}
$$

Here we have used the estimate in (2.11) and (2.13).

The estimate on $\partial_{t} V^{\tau}$ follows by (2.7),

$$
\int_{0}^{T}\left\|\partial_{t} V^{\tau}(t)\right\|_{\Gamma_{G}}^{2} d t=\sum_{n=1}^{N} \int_{t_{n-1}}^{t_{n}}\left\|\frac{v^{n}-v^{n-1}}{\tau}\right\|_{\Gamma_{G}}^{2} d t \leq C N \tau=C T .
$$

Finally, for estimating $\partial_{t} U^{\tau}$ we notice that

$$
\left(\partial_{t} U^{\tau}(t), \phi\right)=\left(\frac{u^{n}-u^{n-1}}{\tau}, \phi\right),
$$

for all $\phi \in H_{0, \Gamma_{D}}^{1}(\Omega)$ and all $t \in\left(t_{n-1}, t_{n}\right]$. By (2.2) this gives

$$
\begin{gathered}
\left|\left(\partial_{t} U^{\tau}, \phi\right)\right| \leq D\left\|\nabla u^{n}\right\|_{\Omega}\|\nabla \phi\|_{\Omega}+M_{q}\left\|\nabla u^{n}\right\|_{\Omega}\|\phi\|_{\Omega} \\
+\frac{\epsilon \tilde{n}}{\tau}\left\|v^{n}-v^{n-1}\right\|_{\Gamma_{G}}\|\phi\|_{\Gamma_{G}} .
\end{gathered}
$$

Using the trace theorem we obtain

$$
\begin{aligned}
\left|\left(\partial_{t} U^{\tau}, \phi\right)\right| \leq & \left(D+M_{q}\right)\left\|\nabla u^{n}\right\|_{\Omega}\|\phi\|_{H^{1}(\Omega)} \\
& +C(\Omega) \frac{\epsilon \tilde{n}}{\tau}\left\|v^{n}-v^{n-1}\right\|_{\Gamma_{G}}\|\phi\|_{H^{1}(\Omega)},
\end{aligned}
$$

for any $\phi \in H_{0, \Gamma_{D}}^{1}(\Omega)$. This implies that

$$
\left\|\partial_{t} U^{\tau}(t)\right\|_{H^{-1}(\Omega)} \leq C\left(\left\|\nabla u^{n}\right\|_{\Omega}+\frac{\epsilon \tilde{n}}{\tau}\left\|v^{n}-v^{n-1}\right\|_{\Gamma_{G}}\right),
$$

for all $t \in\left(t_{n-1}, t_{n}\right]$, and the remaining part is a direct consequence of the $L^{\infty}$ and stability estimates. 
Remark 2.14. For an $\epsilon$-periodic medium, the estimates in Lemma 2.13 can be made $\epsilon$-independent. This follows from the remarks 2.10 and 2.12. In this case the estimates (2.24) and (2.25) become

$$
\begin{aligned}
\left\|U^{\tau}(t)\right\|_{\Omega}+\left\|\partial_{t} U^{\tau}\right\|_{L^{2}\left(0, T ; H^{-1}(\Omega)\right)}^{2}+\left\|\nabla U^{\tau}\right\|_{\Omega^{T}}^{2} & \\
+\epsilon\left(\left\|V^{\tau}(t)\right\|_{\Gamma_{G}}^{2}+\left\|\partial_{t} V^{\tau}\right\|_{\Gamma_{G}^{T}}^{2}\right) & \leq C,
\end{aligned}
$$

for all $0 \leq t \leq T$, where $C$ does not depend on $\tau, \delta$, or $\varepsilon$.

Having the $\tau$ and $\delta$ uniform estimates in Lemma 2.13, we can proceed by sending $\tau$ and $\delta$ to 0 . For any $\tau>0$ and $\delta \geq \tau D_{a}$ we have $\left(U^{\tau}, V^{\tau}, W^{\tau}\right) \in \mathcal{U} \times \mathcal{V} \times L^{\infty}\left(\Gamma_{G}^{T}\right)$. As stated in Remark 2.1, $\delta$ should also satisfy $\delta=o\left(\tau^{\alpha}\right)$ with some $\alpha \in(0,1)$, the reasons for this being made clear later in this paragraph. From now one we assume that both restrictions are satisfied. Then obviously $\tau \searrow 0$ implies the same for $\delta$. Compactness arguments give the existence of a triple $(u, v, w) \in \mathcal{U} \times \mathcal{V} \times L^{\infty}\left(\Gamma_{G}^{T}\right)$ and a subsequence $\tau \searrow 0$, such that
a) $U^{\tau} \rightarrow u$
weakly in
$L^{2}\left((0, T) ; H_{0, \Gamma_{D}}^{1}(\Omega)\right)$,
b) $\partial_{t} U^{\tau} \rightarrow \partial_{t} u$
weakly in
$L^{2}\left((0, T) ; H^{-1}(\Omega)\right)$,
c) $V^{\tau} \rightarrow v$
weakly in
$L^{2}\left((0, T) ; L^{2}\left(\Gamma_{G}\right)\right)$
d) $\partial_{t} V^{\tau} \rightarrow \partial_{t} v$
weakly in
$L^{2}\left((0, T) ; L^{2}\left(\Gamma_{G}\right)\right)$,
e) $W^{\tau} \rightarrow w$
weakly-star in $L^{\infty}\left(\Gamma_{G}^{T}\right)$.

It remains to show that the limit triple $(u, v, w)$ solves (1.1)-(1.2) weakly. This is done in the following.

Theorem 2.15. The limit triple $(u, v, w)$ is a weak solution of (1.1)(1.2) in the sense of Definition 1.1. Moreover, for the dissolution rate we have

$$
w=r(u) \quad \text { a.e. in }\{v=0\} \cap \Gamma_{G}^{T} \text {. }
$$

Proof. By the weak convergence, all the estimates stated in Lemma 2.13 hold for the limit triple $(u, v, w)$. Furthermore, for any $t \in\left(t_{n-1}, t_{n}\right]$, by (2.2) we have

$$
\begin{gathered}
\left(\partial_{t} U^{\tau}(t), \phi\right)_{\Omega}+D\left(\nabla U^{\tau}(t), \nabla \phi\right)_{\Omega} \\
+\left(\nabla \cdot\left(\vec{q} U^{\tau}(t)\right), \phi\right)_{\Omega}+\epsilon \tilde{n}\left(\partial_{t} V^{\tau}(t), \phi\right)_{\Gamma_{G}} \\
=D\left(\nabla\left(U^{\tau}(t)-u^{n}\right), \nabla \phi\right)_{\Omega}+\left(\nabla \cdot\left(\vec{q}\left(U^{\tau}(t)-u^{n}\right)\right), \phi\right)_{\Omega},
\end{gathered}
$$


for all $\phi \in H_{0, \Gamma_{D}}^{1}(\Omega)$. Denoting the terms on the right by $I_{1}(t)$ and $I_{2}(t)$, taking $\phi \in L^{2}\left(0, T ; H_{0, \Gamma_{D}}^{1}(\Omega)\right)$, and integrating (2.28) in time gives

$$
\begin{gathered}
\left(\partial_{t} U^{\tau}, \phi\right)_{\Omega^{T}}+D\left(\nabla U^{\tau}, \nabla \phi\right)_{\Omega^{T}} \\
+\left(\nabla \cdot\left(\vec{q} U^{\tau}\right), \phi\right)_{\Omega^{T}}+\epsilon \tilde{n}\left(\partial_{t} V^{\tau}, \phi\right)_{\Gamma_{G}^{T}} \\
=\sum_{n=1}^{N} \int_{t_{n-1}}^{t^{n}} I_{1}(t)+I_{2}(t) d t .
\end{gathered}
$$

The definition (2.20) of $U^{\tau}$ implies for almost all $0 \leq t \leq T$

$$
\left|I_{1}(t)\right| \leq D \frac{t_{n}-t}{\tau}\left\|\nabla\left(u^{n}-u^{n-1}\right)\right\|_{\Omega}\|\nabla \phi\|_{\Omega},
$$

respectively

$$
\left|I_{2}(t)\right| \leq M_{q} \frac{t_{n}-t}{\tau}\left\|u^{n}-u^{n-1}\right\|_{\Omega}\|\nabla \phi\|_{\Omega} .
$$

In (2.31) we have integrated by parts and used the essential bounds (1.4) on $\vec{q}$. Now we can proceed by estimating the terms on the right in $(2.29)$. For $I_{1}$ we get

$$
\begin{aligned}
\mid \sum_{n=1}^{N} & \int_{t_{n-1}}^{t^{n}} I_{1}(t) d t \mid \leq \sum_{n=1}^{N} \int_{t_{n-1}}^{t^{n}} \frac{t_{n}-t}{\tau} D\left\|\nabla\left(u^{n}-u^{n-1}\right)\right\|_{\Omega}\|\nabla \phi(t)\|_{\Omega} d t \\
& \leq \sum_{n=1}^{N}\left(\frac{\tau}{3} D^{2}\left\|\nabla\left(u^{n}-u^{n-1}\right)\right\|_{\Omega}^{2}\right)^{\frac{1}{2}}\left(\int_{t_{n-1}}^{t^{n}}\|\nabla \phi(t)\|_{\Omega}^{2} d t\right)^{\frac{1}{2}} \\
& \leq \frac{1}{2 \sqrt{3}} \tau^{\frac{1}{4}} \int_{0}^{T}\|\nabla \phi(t)\|_{\Omega}^{2} d t+\frac{\tau^{\frac{3}{4}}}{2 \sqrt{3}} \sum_{n=1}^{N} D^{2}\left\|\nabla\left(u^{n}-u^{n-1}\right)\right\|_{\Omega}^{2} \\
& \leq \frac{1}{2 \sqrt{3}}\left(\int_{0}^{T}\|\nabla \phi(t)\|_{\Omega}^{2} d t+C\right) \tau^{\frac{1}{4}} .
\end{aligned}
$$

In the above we have use the inequality of means (2.17) with $\delta=\tau^{1 / 4}$, as well as the estimates (2.12) and (2.13). In a similar manner, for $I_{2}$ we get

$$
\left|\sum_{n=1}^{N} \int_{t_{n-1}}^{t^{n}} I_{1}(t) d t\right| \leq\left(\frac{1}{2 \sqrt{3}} \int_{0}^{T}\|\nabla \phi(t)\|_{\Omega}^{2} d t+C \tau^{\frac{1}{2}}\right) \tau^{\frac{1}{4}}
$$

Letting $\tau \searrow 0$, the weak convergence of $U^{\tau}$ and $V^{\tau}$, as well as the estimates above imply that $u$ and $v$ satisfy (1.8).

For the dissolution-precipitation equation (1.9) we start by analyzing the behavior of $U^{\tau}$ on $\Gamma_{G}^{T}$. The a-priori estimates, together with Lemma 9 and Corollary 4 of [22], imply

$$
U^{\tau} \rightarrow u \quad \text { strongly in } \quad C\left([0, T] ; H^{-s}(\Omega)\right) \cap L^{2}\left(0, T ; H^{s}(\Omega)\right)
$$

for any $s \in(0,1)$. Then the trace theorem, see for example Satz 8.7 of [26], gives

$$
U^{\tau} \rightarrow u \quad \text { strongly in } \quad L^{2}\left(\Gamma_{G}^{T}\right) .
$$


Taking into account the Lipschitz continuity of $r$, this yields

$$
r\left(U^{\tau}\right) \rightarrow r(u) \quad \text { strongly in } \quad L^{2}\left(\Gamma_{G}^{T}\right)
$$

Further we proceed as for (1.8). For any $t_{n-1}<t \leq t_{n}$ and $\theta \in L^{2}\left(\Gamma_{G}\right)$ we rewrite (2.3) as

$$
\begin{aligned}
\left(\partial_{t} V^{\tau}(t), \theta\right)_{\Gamma_{G}}= & D_{a}\left(r\left(U^{\tau}(t)\right)-W^{\tau}(t), \theta\right)_{\Gamma_{G}} \\
& +D_{a}\left(r\left(u^{n}\right)-r\left(U^{\tau}(t)\right), \theta\right)_{\Gamma_{G}} \\
& +D_{a}\left(W^{\tau}(t)-H_{\delta}\left(v^{n-1}\right), \theta\right)_{\Gamma_{G}}
\end{aligned}
$$

Denoting the last two terms on the right by $I_{3}(t)$ and $I_{4}(t)$, with $\theta \in$ $L^{2}\left(\Gamma_{G}^{T}\right)$, we integrate $(2.32)$ in time and obtain

$$
\begin{aligned}
\left(\partial_{t} V^{\tau}, \theta\right)_{\Gamma_{G}^{T}}= & D_{a}\left(r\left(U^{\tau}\right)-W^{\tau}, \theta\right)_{\Gamma_{G}^{T}} \\
& +\sum_{n=1}^{N} \int_{t_{n-1}}^{t^{n}} I_{3}(t)+I_{4}(t) d t .
\end{aligned}
$$

For almost all $0 \leq t \leq T$, since $r$ and $H_{\delta}$ are Lipschitz, we use the definition of $V^{\tau}$ and $W^{\tau}$ in (2.20) and (2.21) to obtain

$$
\left|I_{3}(t)\right| \leq D_{a} L_{r} \frac{\left(t_{n}-t\right)}{\tau}\left\|u^{n}-u^{n-1}\right\|_{\Gamma_{G}}\|\theta\|_{\Gamma_{G}}
$$

respectively

$$
\left|I_{4}(t)\right| \leq \frac{D_{a}}{\delta} \frac{\left(t_{n}-t\right)}{\tau}\left\|v^{n}-v^{n-1}\right\|_{\Gamma_{G}}\|\theta\|_{\Gamma_{G}}
$$

Using the estimate in (2.14), the first sum in (2.33) is bounded by

$$
\begin{aligned}
& \left|\sum_{n=1}^{N} \int_{t_{n-1}}^{t^{n}} I_{3}(t) d t\right| \leq D_{a} L_{r} \sum_{n=1}^{N} \int_{t_{n-1}}^{t^{n}} \frac{t_{n}-t}{\tau}\left\|u^{n}-u^{n-1}\right\|_{\Gamma_{G}}\|\theta(t)\|_{\Gamma_{G}} d t \\
& \quad \leq D_{a} L_{r} \sum_{n=1}^{N}\left(\frac{\tau}{3}\left\|u^{n}-u^{n-1}\right\|_{\Gamma_{G}}^{2}\right)^{\frac{1}{2}}\left(\int_{t_{n-1}}^{t^{n}}\|\theta(t)\|_{\Gamma_{G}}^{2} d t\right)^{\frac{1}{2}} \\
& \quad \leq \frac{D_{a} L_{r}}{2 \sqrt{3}}\left(\|\theta\|_{\Gamma_{G}^{T}}^{2}+\sum_{n=1}^{N}\left\|u^{n}-u^{n-1}\right\|_{\Gamma_{G}}^{2}\right) \tau^{\frac{1}{2}} \\
& \quad \leq \frac{D_{a} L_{r}}{2 \sqrt{3}}\left(\|\theta\|_{\Gamma_{G}^{T}}^{2}+C\right) \tau^{\frac{1}{2}} .
\end{aligned}
$$


In the above we have use the inequality of means (2.17) with $\delta=\tau^{1 / 2}$. Similarly, for the last sum in (2.33), by (2.7) we get

$$
\begin{aligned}
& \left|\sum_{n=1}^{N} \int_{t_{n-1}}^{t^{n}} I_{4}(t) d t\right| \leq \frac{D_{a}}{\delta} \sum_{n=1}^{N} \int_{t_{n-1}}^{t^{n}} \frac{t_{n}-t}{\tau}\left\|v^{n}-v^{n-1}\right\|_{\Gamma_{G}}\|\theta(t)\|_{\Gamma_{G}} d t \\
& \quad \leq \frac{D_{a}}{\delta} \sum_{n=1}^{N}\left(\frac{\tau}{3}\left\|v^{n}-v^{n-1}\right\|_{\Gamma_{G}}^{2}\right)^{\frac{1}{2}}\left(\int_{t_{n-1}}^{t^{n}}\|\theta(t)\|_{\Gamma_{G}}^{2} d t\right)^{\frac{1}{2}} \\
& \left.\quad \leq \frac{1}{2 \sqrt{3}}\left(\tau D_{a}^{2}\|\theta\|_{\Gamma_{G}^{T}}^{2}+\sum_{n=1}^{N} \| v^{n}-v^{n-1}\right) \|_{\Gamma_{G}}^{2}\right) \frac{1}{\delta} \\
& \quad \leq \frac{D_{a}^{2}}{2 \sqrt{3}}\left(\|\theta\|_{\Gamma_{G}^{T}}^{2}+r\left(M_{u}\right)^{2} \operatorname{meas}\left(\Gamma_{G}\right)\right) \frac{\tau}{\delta} .
\end{aligned}
$$

We let now $\tau \searrow 0$. Since with $\delta=O\left(\tau^{\alpha}\right)$ with $\alpha \in(0,1)$, the above estimates together with the weak convergence of $\partial_{t} V^{\tau}$, the strong convergence of $r\left(U^{\tau}\right)$, as well as the weak-star convergence of $W^{\tau}$ implies that $u, v$ and $w$ satisfy $\left(1.9_{1}\right)$.

It only remains to show that the dissolution rate satisfies $\left(1.9_{2}\right)$. The major impediment is the lacking pointwise $V^{\tau}$ convergence. To resolve this we follow the procedure in Theorem 2.21 of [4]. We define

$$
\underline{V}(t, x):=\liminf _{\tau \searrow 0} V^{\tau}(t, x) \geq 0 \quad \text { a.e. in } \Gamma_{G}^{T},
$$

and decompose $\Gamma_{G}^{T}$ in two subsets defined as

$$
S_{1}=\{\underline{V}>0\} \quad \text { and } \quad S_{2}=\{\underline{V}=0\} .
$$

The equalities should be understood in the almost everywhere sense. Obviously we have $\Gamma_{G}^{T}=S_{1} \cup S_{2}$. As in [4] we show that $v>0$ and $w=1$ in $S_{1}$, while $v=0$ and $w=r(u) \in[0,1]$ in $S_{2}$.

For proving the first assertion we follow exactly the steps in [4], with $\left(V^{\tau}, W^{\tau}\right)$ replacing the pair $\left(v_{\delta}, w_{\delta}\right)$ there. To show the assertion in $S_{2}$ we use the reaction equation (2.33) and rule out the possibility that $v>0$ in $S_{2}$. The weak-star convergence of $W^{\tau}$ implies that $\int_{0}^{t} W^{\tau} \rightarrow$ $\int_{0}^{t} w$ weakly-star in $L^{\infty}\left(\Gamma_{G}^{T}\right)$, and therefore $\lim \inf _{\tau \searrow 0} \int_{0}^{t} W^{\tau} \leq \int_{0}^{t} w$ a.e. in $\Gamma_{G}^{T}$. Further, both $\left(1.9_{1}\right)$ and $(2.33)$ hold in $L^{2}\left(\Gamma_{G}^{T}\right)$ and thus a.e. in $\Gamma_{G}^{T}$. For any $n=1, \ldots, N$ and a.e. $x \in \Gamma_{G}$ and $t \in\left(t_{n-1}, t_{n}\right]$ we integrate the two reaction equations in time and obtain

$$
\begin{aligned}
V^{\tau}= & v_{0}+D_{a}\left(\int_{0}^{t}\left(r\left(U^{\tau}\right)-W^{\tau}\right)+\Sigma_{u}-\Sigma_{w}\right), \\
=v+D_{a}\left(\int_{0}^{t}\left(r\left(U^{\tau}\right)-r(u)\right)\right. & \left.\quad-\int_{0}^{t}\left(W^{\tau}-w\right)+\Sigma_{u}-\Sigma_{w}\right)
\end{aligned}
$$


with

$$
\begin{aligned}
& \Sigma_{u}=\sum_{p=1}^{n-1} \int_{t_{p-1}}^{t_{p}}\left(r\left(u^{p}\right)-r\left(U^{\tau}\right)\right)+\int_{t_{n-1}}^{t}\left(r\left(u^{p}\right)-r\left(U^{\tau}\right)\right), \\
& \left.\Sigma_{w}=\sum_{p=1}^{n-1} \int_{t_{p-1}}^{t_{p}}\left(H_{\delta}\left(v^{p-1}\right)-W^{\tau}\right)\right)+\int_{t_{n-1}}^{t}\left(r\left(u^{p}\right)-r\left(U^{\tau}\right)\right) .
\end{aligned}
$$

We now analyze the $\Sigma$ terms in the above. For a.e $(t, x) \in \Gamma_{G}^{T}$ we have

$$
\begin{aligned}
\left|\Sigma_{u}\right| & \leq L_{r} \sum_{p=1}^{n} \int_{t_{p-1}}^{t_{p}} \frac{t_{p}-t}{\tau}\left|u^{p}-u^{p-1}\right| \leq \frac{L_{r}}{2} \tau\left(n \sum_{p=1}^{n}\left|u^{p}-u^{p-1}\right|^{2}\right)^{\frac{1}{2}} . \\
& \leq \frac{L_{r}}{2}\left(\tau T \sum_{p=1}^{N}\left|u^{p}-u^{p-1}\right|^{2}\right)^{\frac{1}{2}} .
\end{aligned}
$$

By the estimates $(2.14)$, the $L^{2}\left(\Gamma_{G}\right)$ norm of the term on the right vanishes as $\tau \searrow 0$. This also implies the convergence to 0 a.e.

For $\Sigma_{w}$ we obtain analogously

$$
\begin{aligned}
\left|\Sigma_{w}\right| & \leq \frac{\tau}{2 \delta}\left(N \sum_{p=1}^{N}\left|v^{p}-v^{p-1}\right|^{2}\right)^{\frac{1}{2}} \\
& \leq \frac{\tau^{(1+\alpha) / 2}}{2 \delta}\left(\tau^{-\alpha} T \sum_{p=1}^{N}\left|v^{p}-v^{p-1}\right|^{2}\right)^{\frac{1}{2}} .
\end{aligned}
$$

Since $\delta=O\left(\tau^{\alpha}\right)$, the estimates (2.7) imply the pointwise convergence of the right hand side a.e as $\tau \searrow 0$.

With $(t, x) \in S_{2}$, we take the $\liminf _{\tau \backslash 0}$ in (2.36). The $\Sigma$-terms vanish, as seen above. Further, since $U^{\tau}$ converges strongly on $\Gamma_{G}^{T}$ and thus pointwisely, we find

$$
0=v-D_{a} \liminf _{\tau \searrow 0} \int_{0}^{t}\left(W^{\tau}-w\right) \geq v \quad \text { a.e. in } S_{2} .
$$

Therefore $v=0$ in $S_{2}$. Moreover, since $\partial_{t} v \in L^{2}\left(\Gamma_{G}^{T}\right)$, it follows that $\partial_{t} v=0$ a.e. in $S_{2}$, and therefore $w=r(u)$ with $0 \leq w \leq 1$.

Remark 2.16. Besides the convergence of the numerical scheme, Theorem 2.15 also provides an alternative existence proof for a weak solution of (1.1)-(1.2). In [4] this is obtained by fixed point arguments, whereas here the solution is the limit of a time discrete approximating sequence.

\section{A FiXED POINT ITERATION FOR THE TIME DISCRETE PROBLEMS}

In this section we analyze a linear iteration scheme for solving the non-linear time discrete problems $P_{\delta}^{n}$. The nonlinearity appears in the boundary condition (2.3). Numerical experiments based on a Newton type iteration led to instabilities in the form of negative concentrations, 
or to a precipitation in the undersaturated regime $\left(u \leq u^{*}\right)$. Moreover, there is no guarantee of convergence, unless the time stepping is not small enough. Here we discuss an alternative fixed point approach that provides stable results. Moreover, the scheme converges linearly in $H^{1}$, regardless of the initial iteration or of the parameters $\varepsilon, \tau$ and $\delta$.

Assume $u^{n-1}$ and $v^{n-1}$ given and satisfying the bounds in Lemmata 2.3, 2.4, and 2.6. To construct the iteration scheme we proceed as discussed in the beginning of Section 2 and decouple the ion transport equation from the dissolution/precipitation equation on the boundary. Using (2.3), (2.2) can rewritten as

$$
\begin{aligned}
\left(u^{n}-u^{n-1}, \phi\right)_{\Omega} & +\tau D\left(\nabla u^{n}, \nabla \phi\right)_{\Omega}+\tau\left(\nabla \cdot\left(\vec{q} u^{n}\right), \phi\right)_{\Omega} \\
& +\tau \epsilon \tilde{n} D_{a}\left(r\left(u^{n}\right)-H_{\delta}\left(v^{n-1}\right), \theta\right)_{\Gamma_{G}}=0,
\end{aligned}
$$

for all $\phi \in H_{0, \Gamma_{D}}^{1}(\Omega)$. This is a scalar elliptic equation with nonlinear boundary conditions on $\Gamma_{G}$. We first construct a sequence $\left\{u^{n, i}, i \geq 0\right\}$ approximating the solution $u^{n}$ of (3.1). Once this is computed, we use (2.3) for determining $v^{n}$ directly.

Let $L_{r}$ be the Lipschitz constant of the precipitation rate $r$ on the interval $\left[0, M_{u}\right]$. With

$$
\mathcal{K}:=\left\{u \in H_{0, \Gamma_{D}}^{1}(\Omega) / 0 \leq u \leq M_{u} \text { a. e. in } \Omega\right\},
$$

and for a given $u^{n, i-1} \in \mathcal{K}$, we define $u^{n, i}$ as the solution of the linear elliptic equation

$$
\begin{aligned}
\left(u^{n, i}-\right. & \left.u^{n-1}, \phi\right)_{\Omega}+\tau D\left(\nabla u^{n, i}, \nabla \phi\right)_{\Omega}+\tau\left(\nabla\left(\vec{q} u^{n, i}\right), \phi\right)_{\Omega} \\
= & \tau \epsilon \tilde{n} D_{a} L_{r}\left(u^{n, i-1}-u^{n, i}, \phi\right)_{\Gamma_{G}} \\
& -\tau \epsilon \tilde{n} D_{a}\left(r\left(u^{n, i-1}\right)-H_{\delta}\left(v^{n-1}\right), \theta\right)_{\Gamma_{G}},
\end{aligned}
$$

for all $\phi \in H_{0, \Gamma_{D}}^{1}(\Omega)$. The starting point of the iteration can be chosen arbitrarily in $\mathcal{K}$. However, a good initial guess is $u^{n, 0}=u^{n-1}$.

Comparing the above to (3.1), disregarding the superscripts $i-1$ and $i$, the only difference is in the appearance of the first term on the right in (3.3). In the case of convergence, this term vanishes, so $u^{n, i}$ approaches $u^{n}$. Before making this sentence more precise we mention that the above construction is common in the analysis of nonlinear elliptic problems, in particular when sub- or supersolutions are sought (see, e. g., [25], pp. 96). In [20], this approach is placed in a fixed point context, for approximating the solution of an elliptic problem with a nonlinear and possibly unbounded source term (see also [27]). The same ideas are followed in [23] and [19], where similar schemes are considered for the implicit discretization of a degenerate (fast diffusion) 
problem in both conformal and mixed formulation. We also mention here [24] for a related work on nonlinear elliptic equations.

Since (3.3) defines a fixed point iteration, only a linear convergence rate is to be expected. This drawback is compensated by the stability of the approximation sequence, as well as its guaranteed convergence. These statements are made precise below.

Lemma 3.1. Assume $0 \leq u^{n, i-1} \leq M_{u}$ almost everywhere on $\Omega$. Then $u^{n, i}$ solving (3.3) satisfies the same bounds.

Proof. This can be shown by following the ideas used in proving Lemmata 2.3 and 2.4. We omit the details here.

Starting the iteration with $u^{n, 0} \in \mathcal{K}$, a straightforward mathematical induction argument shows the stability of the entire sequence $\left\{u^{n, i}, i \geq\right.$ $0\}$. To prove the convergence of the scheme we let

$$
e^{n, i}:=u^{n}-u^{n, i}
$$

denote the error at iteration $i$ and define the $H^{1}$-equivalent norm

$$
\||f|\|^{2}:=\|f\|_{\Omega}^{2}+\tau D\|\nabla f\|_{\Omega}^{2}+\zeta\|f\|_{\Gamma_{G}}^{2} .
$$

Here $f$ is any function in $H_{0, \Gamma_{D}}^{1}(\Omega)$, and the constant $\zeta>0$ is defined as

$$
\zeta:=\frac{\tau}{2} \epsilon \tilde{n} D_{a} L_{r}
$$

Notice that if $\tau$ is reasonably small, $\zeta<1$.

The lemma below shows that the iteration error defined in (3.4) is a contraction in the norm (3.5).

Lemma 3.2. For $\tau<2 /\left(\epsilon \tilde{n} D_{a} L_{r}\right)$, an $i$-independent constant $0<\gamma<$ 1 exists such that

$$
\left\|\left|e^{n, i}\right|\right\|^{2} \leq \gamma\|\| e^{n, i-1} \|^{2},
$$

provided $u^{n, i-1}$ satisfies the bounds in Lemma 3.1.

Proof. With $\zeta$ given above, we start by adding $2 \zeta\left(u^{n}, \phi\right)_{\Gamma_{G}}$ on both side of (2.2). Subtracting (3.3) from the resulting equation gives

$$
\begin{aligned}
\left(e^{n, i}, \phi\right)_{\Omega} & +\tau D\left(\nabla e^{n, i}, \nabla \phi\right)_{\Omega}-\tau\left(q e^{n, i}, \nabla \phi\right)_{\Omega}+2 \zeta\left(e^{n, i}, \phi\right)_{\Gamma_{G}} \\
& =2 \zeta\left(e^{n, i-1}, \phi\right)_{\Gamma_{G}}+\tau \epsilon \tilde{n} D_{a}\left(r\left(u^{n}\right)-r\left(u^{n, i-1}\right), \phi\right)_{\Gamma_{G}} .
\end{aligned}
$$


Since $\nabla \cdot q=0$ and $e^{n, i}$ has a zero trace on $\Gamma_{D}$, taking $\phi=e^{n, i}$ into above yields

$$
\begin{aligned}
& \left\|e^{n, i}\right\|_{\Omega}^{2}+\tau D\left\|\nabla e^{n, i}\right\|_{\Omega}^{2}+2 \zeta\left\|e^{n, i}\right\|_{\Gamma_{G}}^{2} \\
& \quad \leq \tau \epsilon \tilde{n} D_{a}\left\|L_{r} e^{n, i-1}-\left(r\left(u^{n}\right)-r\left(u^{n, i-1}\right)\right)\right\|_{\Gamma_{G}}\left\|e^{n, i}\right\|_{\Gamma_{G}} \\
& \quad \leq 2 \zeta\left\|e^{n, i-1}\right\|_{\Gamma_{G}}\left\|e^{n, i}\right\|_{\Gamma_{G}} .
\end{aligned}
$$

We then immediately get

$$
\left\|\left|e^{n, i}\left\|\left.\right|^{2} \leq \zeta\right\| e^{n, i-1}\left\|_{\Gamma_{G}}^{2}=\zeta(1-\alpha)\right\| e^{n, i-1}\left\|_{\Gamma_{G}}^{2}+\zeta \alpha\right\| e^{n, i-1} \|_{\Gamma_{G}}^{2},\right.\right.
$$

where $\||\cdot|||$ is introduced in (3.5) and $\alpha$ is an arbitrary constant in $(0,1)$ to be chosen below.

Using the trace estimate (2.16) and the inequality of means, for any $\beta>0$ we have

$$
\left\|e^{n, i-1}\right\|_{\Gamma_{G}}^{2} \leq\left(C_{1}+\frac{C_{2}^{2}}{4 \beta}\right)\left\|e^{n, i-1}\right\|_{\Omega}^{2}+\beta\left\|\nabla e^{n, i-1}\right\|_{\Omega}^{2}
$$

Using this into (3.7) and taking $\alpha \in(0,1), \beta>0$, and $\gamma>0$ satisfying the constraints

$$
\begin{aligned}
\zeta \alpha\left(C_{1}+\frac{C_{2}^{2}}{4 \beta}\right) & \leq \gamma \\
\zeta \alpha \beta & \leq \tau D \gamma \\
\zeta(1-\alpha) & \leq \gamma
\end{aligned}
$$

we obtain

$$
\begin{aligned}
&\left\|e^{n, i}\right\|\left\|^{2} \leq(1-\alpha) \zeta\right\| e^{n, i-1}\left\|_{\Gamma_{G}}^{2}+\alpha \beta \zeta\right\| \nabla e^{n, i-1} \|_{\Omega}^{2} \\
&+\alpha \zeta\left(C_{1}+\frac{C_{2}^{2}}{4 \beta}\right)\left\|e^{n, i-1}\right\|_{\Omega}^{2} \leq \gamma\|\| e^{n, i-1} \|\left.\right|^{2} .
\end{aligned}
$$

With

$$
\beta=\frac{\tau D}{2}\left(C_{1}+\sqrt{C_{1}^{2}+\frac{C_{2}^{2}}{\tau D}}\right), \text { and } \alpha=\left(1+\frac{C_{1}}{2}+\frac{1}{2} \sqrt{C_{1}^{2}+\frac{C_{2}^{2}}{\tau D}}\right)^{-1},
$$

the restrictions on $\alpha$ and $\beta$ are fulfilled. Further, this choice also gives identical lower bounds for $\gamma$ in (3.8), for which we can now take

$$
\gamma=\zeta\left(C_{1}+\sqrt{C_{1}^{2}+\frac{C_{2}^{2}}{\tau D}}\right)\left(2+C_{1}+\sqrt{C_{1}^{2}+\frac{C_{2}^{2}}{\tau D}}\right)^{-1}
$$

By the assumptions on $\tau$ we have $\gamma<1$, hence the iteration error is contractive in the norm defined in (3.5). 
Remark 3.3. The iteration (3.3) defines an operator $\mathcal{T}: \mathcal{K} \rightarrow \mathcal{K}$. Following the steps in the proof of Lemma 3.2, we can show that $\mathcal{T}$ is a contraction with respect to the norm defined in (3.5). Therefore $\mathcal{T}$ has a unique fixed point, yielding the existence and uniqueness of a solution for the nonlinear equation (3.1). This immediately implies the existence and uniqueness of a solution for Problem $\mathrm{P}_{\delta}^{n}$, as stated in Lemma 2.2.

Lemma 3.2 implies the linear convergence in $H^{1}$ of the iteration sequence $\left\{u^{n, i}, i \geq 0\right\}$. Moreover, its limit is the solution $u^{n}$ of (3.1).

Theorem 3.4. With $u^{n, 0} \in H_{0, \Gamma_{D}}^{1}(\Omega)$ bounded essentially by 0 and $M_{u}$, if $\tau<2 /\left(\epsilon \tilde{n} D_{a} L_{r}\right)$, the iteration (3.3) is convergent. Specifically, for all $i>0$ we have

$$
\left\|\left|e^{n, i}\right|\right\|^{2} \leq \gamma^{i}\left|\left\|e^{n, 0} \mid\right\|^{2} .\right.
$$

Remark 3.5. The contraction constant $\gamma$ in the above is bounded from above by $\zeta=\tau \epsilon \tilde{n} D_{a} L_{r} / 2$. Notice that the first term in the $H^{1}$ equivalent norm in (3.5) does not depend on $\tau$. As $\tau \searrow 0, \gamma$ approaches 0 , implying a fast convergence of the iteration at least in $L^{2}$ sense. In the numerical computations presented in the following section, 3 to 4 iterations were enough for obtaining a good numerical approximation of the time discrete solution.

As stated in Theorem 3.4, convergence is achieved as $i \rightarrow \infty$. In practice we stop this procedure after a finite (small) number of iterations. This means that at each time step we are adding an iteration error to the time discrete approximation. This error depends on the number of iterations performed per time step, as well as on the initial iteration error. As mentioned before, the solution at the previous time step can be used for initiating the iteration. In the remaining part of this section we show that by this choice the total error is vanishing as $\tau \searrow 0$.

To make this statement rigorous we assume that $i$ iterations are performed at each time step $n$. The computed solution $\tilde{u}^{n}=u^{n, i}$ will only be an approximation of $u^{n}$, the solution of (3.1). Let now $\tilde{e}^{n}$ denote the error at the time step $n$,

$$
\tilde{e}^{n}:=u^{n}-\tilde{u}^{n} .
$$

Based on the stability estimates in Lemma 2.11, we can estimate the total error that is accumulated in the numerical approximation of the time discrete sequence $\left\{u^{n}, n=1, \ldots, N\right\}$. 
Lemma 3.6. Assume that, for each $n=1, \ldots, N$, $i$ iterations (3.3) are performed by starting with $u^{n, 0}=\tilde{u}^{n-1}=u^{n-1, i}$. We have

$$
\sum_{n=1}^{N}\left\|\tilde{e}^{n}\right\| \leq \leq \frac{\gamma^{i / 2}}{\tau^{1 / 4}}
$$

Remark 3.7. Since $\gamma=O(\tau)$, the total error vanishes as $\tau \searrow 0$.

Proof. With $u^{n, 0}=\tilde{u}^{n-1}$, the initial error at the time step $n$ is given by

$$
e^{n, 0}=u^{n}-\tilde{u}^{n-1}=u^{n}-u^{n-1}+\tilde{e}^{n-1} .
$$

By Theorem 3.4 the error at the time step $n$ can be estimated as

$$
\| \tilde{e}^{n}||\left|\leq \gamma^{i / 2}\right||| e^{n, 0}|| \mid \leq \gamma^{i / 2}\left(||\left|u^{n}-u^{n-1}\right|\|+\| \tilde{e}^{n-1}|| \mid\right) .
$$

Repeating this estimates inductively for $n=1, \ldots, N$, since $\tilde{e}^{0}=0$ we obtain

$$
\left\|\left|\tilde{e}^{n}\right|\right\| \leq \sum_{k=1}^{n} \gamma^{(n+1-k) i / 2}||\left|u^{k}-u^{k-1}\right| \| .
$$

Adding the above for $n=1$ up to $N$ gives

$$
\sum_{n=1}^{N}\left\|\left|\tilde { e } ^ { n } \left\|\left|\leq \frac{\gamma^{i / 2}}{1-\gamma^{i / 2}} \sum_{k=1}^{N}\left(1-\gamma^{(N+1-k) i / 2}\right)\left\|\left|u^{k}-u^{k-1}\right|\right\| .\right.\right.\right.\right.
$$

By the definition of $\gamma$ in (3.10), if $\tau$ is small enough the fraction in the above can be bounded by $C \gamma^{i / 2}$ for some constant $C>0$. Now the proof can be completed straightforwardly by applying the stability estimates in Lemma 2.11.

\section{NUMERICAL RESUlts}

In this section we present some numerical simulations obtained for the undersaturated regime, when only dissolution is possible. Extensive numerical results for both dissolution and precipitation, and for high or low Damköhler numbers, will be presented in a forthcoming paper. All the computations are implemented in the research software SciFEM (Scilab Finite Element Method ${ }^{1}$, see also [2]).

Here we consider a two dimensional domain $\Omega$, obtained by removing the disc $B_{R}(0,0)$ of radius $R=1 / 4$ and centered in the origin form the square $(-1 / 2,1 / 2)^{2}$. The impermeable grain is represented by the disc, and flow takes place around the grain, from the left boundary to the right one. The present experiments are similar to those presented in [4], where a simple geometry - a two dimensional strip - has been

\footnotetext{
${ }^{1}$ See http://lapcs.univ-lyon1.fr/ ${ }^{\sim}$ clopeau/scilab/index.html
} 
considered. There the occurrence of a dissolution front has been investigated both analytically and numerically. After a waiting time $t^{*}$, the front started moving in the flow direction. As we will see below, the present computations are revealing similar features.

We have used the following parameters and rate function:

$$
D=1, D_{a}=1, \varepsilon=1, \tilde{m}=\tilde{n}=1.0 \text {, and } r(u, c)=\frac{10}{9}[u]_{+}[u-0.1]_{+} .
$$

This gives $u_{*}=0.1$ and $u^{*}=1.0$. The initial and (external) boundary conditions are

$$
\begin{aligned}
v_{I} & =0.1, & & \text { on } \Gamma_{G}, \\
u_{I} & =1.0, & & \text { in } \Omega, \\
u & =u_{*}, & & \text { if } x=-1 / 2, t>0, \\
\partial_{\nu} u & =0, & & \text { on } \partial \Omega \backslash\left\{\Gamma_{G} \cup\{x=-1 / 2\}\right\} .
\end{aligned}
$$

With the data above, the system is initially in equilibrium: no precipitation or dissolution is taking place. This equilibrium is perturbed by injecting an undersaturated fluid at the inflow boundary $x=-1 / 2$. We start with a uniformly distributed layer of precipitate. As resulting from the the $L^{\infty}$ estimates, only dissolution is possible.

The fluid velocity $\vec{q}$ is determined by solving numerically the Stokes system

$$
\begin{cases}\mu \triangle \vec{q}=\nabla p, & \text { in } \Omega, \\ \nabla \cdot \vec{q}=0, & \text { in } \Omega .\end{cases}
$$

where $\mu=0.01$. At both the in- and outflow boundaries $x= \pm 1 / 2$ we take $\vec{q}=(2,0)$. On the upper and lower boundaries $y= \pm 1 / 2$ we take $q_{y}=0$, and $\partial_{y} q_{x}=0$. For symmetry reasons, the computations are restricted to the upper half of the domain. The numerical approximation of $\vec{q}$ is obtained by employing the bubble stabilized finite element method proposed in [21] (see also [13]). The computed velocity field is presented in Figure 1.

For computing the cation concentration $u$ and the precipitate concentration $v$ we have applied the the scheme (2.2)-(2.3) with a fixed time step $\tau=10^{-4}$, and the regularization parameter $\delta=10^{-4}$. As mentioned in the appendix of [3] (see also Theorem 2.15), we set $w=\min \{r(u), 1\}$ whenever $v=0$. The emerging nonlinear time discrete problems are solved by the linearization (3.3), with $u^{n, 0}=u^{n-1}$. The procedure is stopped once the maximal difference between two successive iterations is reduced below $10^{-3}$. In our experiments 3 to 4 iterates were sufficient to fulfill this stopping criterion. We have used piecewise linear finite elements for the spatial discretization of the time discrete problems. 


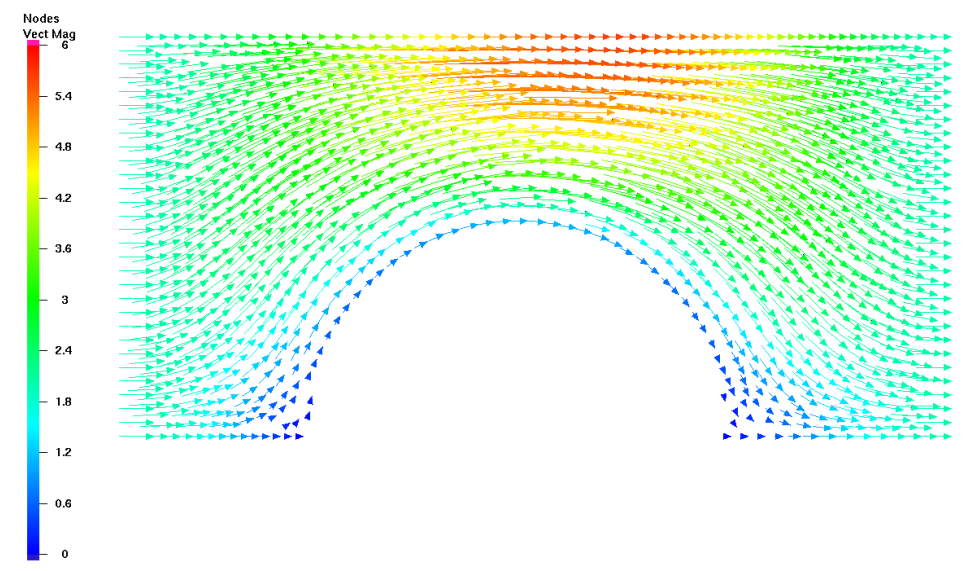

FiguRE 1 . The computed velocity field $\vec{q}$
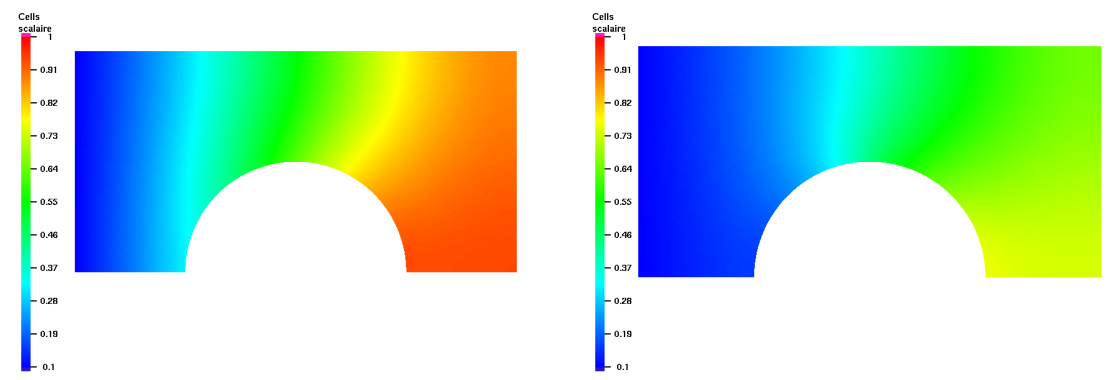

Figure 2. Concentration of the cation $u$ at $t_{1}=0.1$ (left) and $t_{2}=0.2$ (right)

The computations are performed up to $T=0.4$. Figure 2 is displaying the cation concentration $u$ at two different times, $t_{1}=0.1$ and $t_{2}=0.2$. As expected, $u$ is higher at the outflow than at the inflow, and stays bounded by $u_{*}$ and $u^{*}$. This excludes the possibility of precipitation. Moreover, the amount of cations is decreasing in time. Figure 3 displays the precipitate concentration $v$ as a function of the arc length $s=R \theta$. Here $R=1 / 4$, while $\theta$ is the angle between the radii through the leftmost point $(-R, 0)$, respectively through the location of interest on $\Gamma_{G}$. Notice that $v$ is monotone with respect to $s$. A major difference can be noticed in the $v$ profiles for $t_{1}=0.1$ and $t_{2}=0.2$. In the former case we have $v>0$ for any $s$, thus precipitate is present everywhere. 

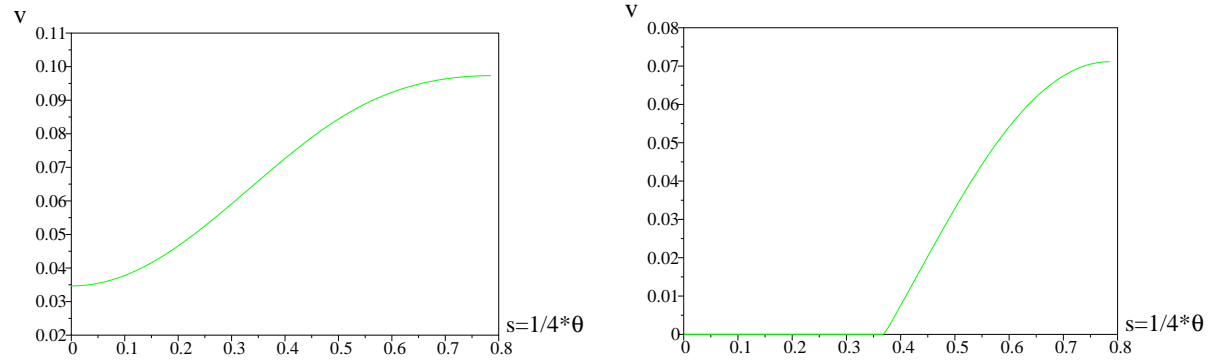

Figure 3. Concentration of the precipitate $v$ at $t_{1}=0.1$ (left) and $t_{2}=0.2$ (right) as a function of the arc length

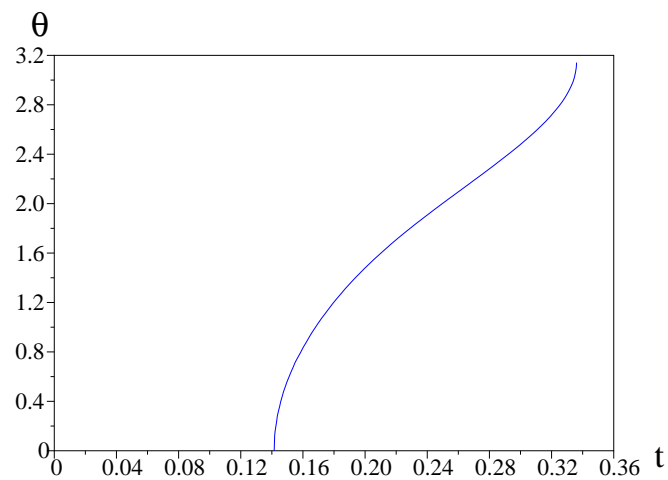

Figure 4. The position of the dissolution front as a function of time: at $t^{*} \approx 0.1415$ precipitate is present everywhere on the grain boundary; after $\tilde{t} \approx 0.3359$ the entire precipitate has bee dissolved

For $t_{2}$ we identify two different regions on $\Gamma_{G}$. In the left one the precipitate has been completely dissolved, whereas crystals can be found everywhere in the region on the right. This shows the occurrence of a dissolution front, located at the free boundary separating the two regions on $\Gamma_{G}$. At $t_{2}$ the dissolution front is located close to $(0, R)$, the upper point of $\Gamma_{G}$.

The evolution of the dissolution front is presented in Figure 4, where the angle $\theta$ is given as a function of time. Up to $t^{*} \approx 0.1415$, the precipitate is present everywhere on the grain surface $\Gamma_{G}$. For any time between $t^{*}$ and $\tilde{t} \approx 0.3359$ the dissolution front moves towards the rightmost point on $\Gamma_{G},(R, 0)$. After $\tilde{t}$ the entire precipitate has been dissolved. Notice the fast movement of the free boundary as $t$ greater than but close to the waiting time $t^{*}$. This is due to the fact 
that the grain boundary $\Gamma_{G}$ is nearly transversal to the flow. Since the inflow boundary conditions for $u$ and $\vec{q}$ are constant, a minimal variation of $u$ in the vertical direction can be expected. This implies a similar behavior for $v$ if $s$ ( or $\theta$ ) is close to 0 , and therefore the complete dissolution along this region occurs nearly simultaneously.

\section{CONCLUSiON}

We have analyzed a numerical scheme for the time discretization of (1.1)-(1.2). This is a simplification of the pore scale model for crystal dissolution and precipitation in porous media. The main difficulties associated with the model, a parabolic problem that is coupled through the boundary to a differential inclusion, are still present in this setting.

The numerical scheme is implicit in $u$ and explicit in $v$. A regularization step is employed for dealing with the multivalued dissolution rate. We prove the stability of the scheme in both $L^{\infty}$, as well as energy norms. Further, compactness arguments are used for showing that the scheme is convergent.

A fixed point iteration is proposed for solving the nonlinear time discrete problems. This linearization is stable and converges linearly, regardless of the discretization parameters and the starting point.

Acknowledgment The work of C.J. van Duijn and I.S. Pop was supported by the Dutch government through the national program BSIK: knowledge and research capacity, in the ICT project BRICKS (http://www.bsik-bricks.nl), theme MSV1. This research was initiated while V. Devigne spent six months at the Technische Universiteit Eindhoven, supported through an European Community Marie Curie Fellowship (contract number HPMT-CT-2001-00422). We would like to thank Prof. A. Mikelić (Lyon) and to the members of the Applied Analysis group in Eindhoven for their suggestions.

\section{REFERENCES}

[1] N. Bouillard, R. Eymard, R. Herbin, Ph. Montarnal, Diffusion with dissolution and precipitation in a porous media: mathematical analysis and numerical approximation of a simplified model, submitted.

[2] V. M. Devigne, Ecoulements et Conditions aux Limites Particulires Appliquées en Hydrogéologie et Théorie Mathématique des Processus de Dissolution/Précipitation en Milieux Poreaux. Ph'D Thesis, Université Lyon 1 (2006).

[3] C. J. van Duijn, P. Knabner, Travelling wave behaviour of crystal dissolution in porous media flow, European J. Appl. Math. 8 (1997), 49-72.

[4] C. J. van Duijn, I. S. Pop, Crystal dissolution and precipitation in porous media : pore scale analysis, J. Reine Angew. Math. 577 (2004), 171-211. 
[5] R. Eymard, T. Gallout, R. Herbin, D. Hilhorst, M. Mainguy, Instantaneous and noninstantaneous dissolution: approximation by the finite volume method, in Actes du 30ème Congrès d'Analyse Numérique: CANum '98 (Arles, 1998), R. Boyer et al. (Eds.), ESAIM Proc. 6, Soc. Math. Appl. Indust., Paris (1999), 41-55.

[6] B. Faugeras, J. Pousin, F. Fontvieille, An efficient numerical scheme for precise time integration of a diffusion-dissolution/precipitation chemical system, Math. Comp. 75 (2006), 209-222.

[7] P. Grisvard, Elliptic Problems in Nonsmooth Domains, Monographs and Studies in Mathematics 24, Pitman (Advanced Publishing Program), Boston, MA (1985).

[8] U. Hornung, W. Jäger, A model for chemical reactions in porous media, in Complex Chemical Reaction Systems. Mathematical Modeling and Simulation, J. Warnatz, W. Jäger (Eds.), Chemical Physics 47, Springer, Berlin, (1987).

[9] U. Hornung, W. Jäger, Diffusion, convection, adsorption, and reaction of chemicals in porous media, J. Differential Equations 92 (2001), 199-225.

[10] U. Hornung, W. Jäger, A. Mikelić, Reactive transport through an array of cells with semipermeable membranes, RAIRO Modél. Math. Anal. Numér., 28 (1994), 59-94.

[11] R. B. Kellogg, J. E. Osborn, A regularity result for the Stokes problem in a convex polygon, J. Functional Analysis 21 (1976), 397-431.

[12] P. Knabner, C. J. van Duijn, S. Hengst, An analysis of crystal dissolution fronts in flows through porous media. Part 1: Compatible boundary conditions, Adv. Water Res. 18 (1995), 171-185.

[13] P. Knobloch, On the application of the $p_{1}^{\text {mod }}$ element to incompressible flow problems, Comput. Vis. Sci. 6 (2004), 185-195.

[14] J. R. Kweona, R. B. Kellogg, Compressible Stokes problem on nonconvex polygonal domains, J. Differential Equations 176 (2001), 290-314.

[15] E. Maisse, J. Pousin, Diffusion and dissolution/precipitation in an open porous reactive medium, J. Comput. Appl. Math. 82 (1997), 279-290.

[16] E. Maisse, J. Pousin, Finite element approximation of mass transfer in a porous medium with non equilibrium phase change, J. Numer. Math. 12 (2004), 207231.

[17] A. Mikelić, V. Devigne, C. J. van Duijn, Rigorous upscaling of the reactive flow through a pore, under dominant Peclet and Damkohler numbers, CASA Report 05-19, Eindhoven University of Technology (2005).

[18] I. S. Pop, V. Devigne, C. J. van Duijn, T. Clopeau, A numerical scheme for the micro scale dissolution and precipitation in porous media, in Numerical Mathematics and Advanced Applications, A. Bermudez de Castro, D. Gomez, P. Quintela, P. Salgado (Eds.), Springer-Verlag Heidelberg, 2006, pp. 349 358.

[19] I. S. Pop, F. Radu, P. Knabner, Mixed finite elements for the Richards' equation: linearization procedure, J. Comput. Appl. Math. 168 (2004) 365-373.

[20] I. S. Pop, W. A. Yong, On the existence and uniqueness of a solution for an elliptic problem, Studia Univ. Babeş-Bolyai Math. 45 (2000), 97-107.

[21] A. Russo, Bubble stabilization of finite element methods for the linearized incompressible Navier-Stokes equations, Comput. Methods appl. Mech. Engrg. 132 (1996), 335-343. 
[22] J. Simon, Compact sets in the space $L^{p}(0, T ; B)$, Ann. Mat. Pura Appl.(4) 146 (1987), $65-96$.

[23] M. Slodička, A robust and efficient linearization scheme for doubly nonlinear and degenerate parabolic problems arising in flow in porous media, SIAM J. Sci. Comput. 23 (2002), 1593-1614.

[24] M. Slodička, A robust linearisation scheme for a nonlinear elliptic boundary value problem: error estimates, ANZIAM J. 46 (2005), 449-470.

[25] J. Smoller, Shock Waves and Reaction-Diffusion Equations, Springer Verlag, New York, 1983.

[26] J. Wloka, Partielle Differentialgleichungen, B. G. Teubner, Stuttgart (1982).

[27] W. A. Yong, I. S. Pop, A numerical approach to porous medium equations, Preprint 96-50 (SFB 359), IWR, University of Heidelberg (1996).

\author{
$\square$ Centre SITE
}

Ecole Nationale Supérieure des Mines de Saint-Etienne 158, Cours Fauriel, 4227 St-Etienne cedex 02, FRANCE e-mail: Vincent.Devigne@emse.fr

$\sharp$ Department of Mathematics and Computing Science Eindhoven University of Technology

P.O. Box 513, 5600 MB Eindhoven, The Netherlands e-mail: \{C.J.v.Duijn, I.Pop\}@tue.nl

b Institut Camille Jordan

Université Claude Bernard Lyon I, site de Gerland Bât A, bur. 1304 50 , Av. Tony Garnier, 69367 Lyon Cedex 07, FRANCE e-mail: \{Thierry.Clopeau,Vincent.Devigne\}@univ-lyon1.fr 